\title{
Evolution of switchable aposematism: insights from individual-based simulations
}

\author{
Woncheol Song $^{1}$, Sang-im Lee ${ }^{\text {Corresp., }}{ }^{2}$, Piotr G Jablonski ${ }^{\text {Corresp. } 1,3}$ \\ ${ }^{1}$ School of Biological Sciences, Seoul National University, Seoul, South Korea \\ 2 School of Undergraduate Studies, Daegu-Gyeongbuk Institute of Science and Technology, Daegu, South Korea \\ 3 Museum and Institute of Zoology, Polish Academy of Sciences, Warsaw, Poland \\ Corresponding Authors: Sang-im Lee, Piotr G Jablonski \\ Email address: sangim@dgist.ac.kr, piotrjab@behecolpiotrsangim.org
}

Some defended prey animals can switch on their normally hidden aposematic signals. This switching may occur in reaction to predators' approach (pre-attack signals) or attack (postattack signals). Switchable aposematism has been relatively poorly studied, but we can expect that it might bring a variety of benefits to an aposmetic organism. First, the switching could startle the predators (deimatism). Second, it could facilitate aversive learning. Third, it could minimize exposure or energetic expense, as the signal can be switched off. These potential benefits might offset costs of developing, maintaining and utilizing the switchable traits. Here we focused on the third benefit of switchability, the cost-saving aspect, and developed an individual-based computer simulation of predators and prey. In 88128 model runs, we observed evolution of permanent, pre-attack, or postattack aposematic signals of varying strength. We found that, in general, the pre-attack switchable aposematism may require moderate predator learning speed, high basal detectability, and moderate to high signal cost. On the other hand, the post-attack signals may arise under slow predator learning, low basal detectability, and high signal cost. When predator population turnover is fast, it may lead to evolution of post-attack aposematic signals that are not conforming to the above tendency. We also suggest that a high switching cost may exert different selection pressure on the pre-attack than the postattack switchable strategies. To our knowledge, these are the first theoretical attempts to systematically explore the evolution of switchable aposematism relative to permanent aposematism in defended prey. Our simulation model is capable of addressing additional questions beyond the scope of this article, and we open the simulation software, program manual, and source code for free public use. 


\section{Evolution of switchable aposematism: insights from} 2 individual-based simulations

3

4
Woncheol Song ${ }^{1}$, Sang-im Lee ${ }^{2}$, Piotr G. Jablonski ${ }^{1,3}$

${ }^{1}$ Laboratory of Behavioral Ecology and Evolution, School of Biological Sciences, Seoul National University, Seoul, South Korea

${ }^{2}$ School of Undergraduate Studies, Daegu-Gyeongbuk Institute of Science and Technology, Daegu, South Korea.

${ }^{3}$ Museum and Institute of Zoology, Polish Academy of Sciences, Warsaw, Poland

Corresponding Authors:

Sang-im Lee ${ }^{2}$

School of Undergraduate Studies, Daegu-Gyeongbuk Institute of Science and Technology, Daegu 42988, South Korea

Email address: sangim@dgist.ac.kr

Piotr G. Jablonski ${ }^{1,3}$

Laboratory of Behavioral Ecology and Evolution, School of Biological Sciences, Seoul National University, Seoul 08826, South Korea

Email address: piotrjab@behecolpiotrsangim.org 


\section{Abstract}

44

45

46

47

48

49

50

51

52

53

54

55

56

57

58

59

60

61

62

63

64

65

66

67

68

69

70

71

72

73

74

75

76

77

78

79

80

81

Some defended prey animals can switch on their normally hidden aposematic signals. This switching may occur in reaction to predators' approach (pre-attack signals) or attack (post-attack signals). Switchable aposematism has been relatively poorly studied, but we can expect that it might bring a variety of benefits to an aposmetic organism. First, the switching could startle the predators (deimatism). Second, it could facilitate aversive learning. Third, it could minimize exposure or energetic expense, as the signal can be switched off. These potential benefits might offset costs of developing, maintaining and utilizing the switchable traits. Here we focused on the third benefit of switchability, the cost-saving aspect, and developed an individual-based computer simulation of predators and prey. In 88128 model runs, we observed evolution of permanent, pre-attack, or post-attack aposematic signals of varying strength. We found that, in general, the pre-attack switchable aposematism may require moderate predator learning speed, high basal detectability, and moderate to high signal cost. On the other hand, the post-attack signals may arise under slow predator learning, low basal detectability, and high signal cost. When predator population turnover is fast, it may lead to evolution of post-attack aposematic signals that are not conforming to the above tendency. We also suggest that a high switching cost may exert different selection pressure on the pre-attack than the post-attack switchable strategies. To our knowledge, these are the first theoretical attempts to systematically explore the evolution of switchable aposematism relative to permanent aposematism in defended prey. Our simulation model is capable of addressing additional questions beyond the scope of this article, and we open the simulation software, program manual, and source code for free public use.

\section{Introduction}

\section{Switchable aposematism.}

Some prey animals possess defensive measures that make them unprofitable to predators. Such defended prey often signals their unpalatability via bright colors, alarming sounds, or other conspicuous components. These signals can affect predator's decision to ingest the prey because the predator learns association between the signal and the prey unprofitability. This form of antipredatory strategy is called aposematism. Evolutionary biologists have explored various dimensions of aposematism, but its behavioral aspect had not received adequate attention until recent years. While some aposematic signals such as permanent coloration are fixed and operate continuously, other signals can be behaviorally controlled, by sound generation (Dowdy \& Conner 2016), wing movement (Kang et al. 2016), bioluminescence (De Cock \& Matthysen 1999), physiological color change (Umbers et al. 2014), or postural change (Lariviere \& Messier 1996). We call this form "switchable aposematism."

Peer) reviewing PDF | (2019:09:41588:2:0:NEW 2 Mar 2020) 
82

83

84

85

86

87

88

89

90

91

92

93

94

95

96

97

98

99

100

101

102

103

104

105

106

107

108

109

110

111

112

113

114

115

116

117

118

119

120

Historically, switchable aposematism has been described with various adjectives such as "facultative" (Sivinski 1981; Grober 1988), "post-attack" (Umbers \& Mappes 2015; Kang et al. 2016), "early-acting" (Broom et al. 2010), "deimatic" (Umbers et al. 2015; Umbers \& Mappes 2015), or "switchable" (Umbers et al. 2017), implying various functional or temporal characteristics found in each study species. This had led to disputes regarding the use of "deimatic" as either descriptor of function or that of a form (Umbers et al. 2015; Umbers \& Mappes 2015; Skelhorn et al. 2016; Umbers et al. 2017). In this article, we chose to use a term that indisputably concerns the form of the display behavior: 'switchable.'

\section{The renewed interest in switchable aposematism.}

In contrast to the well-developed knowledge on switchable displays in undefended (profitable) prey in the context of "startling" or "deimatic" function (sudden display surprising the predator away: Edmunds (1972); Schlenoff (1985); Grandcolas \& Desutter-Grandcolas (1998); Vallin et al. (2005); Bura et al. (2011)), switchable aposematic signals of defended prey have been largely ignored. Notable exceptions are early studies on bioluminescence in defended prey (Sivinski 1981; Grober 1988) and short descriptions of switchable conspicuous signals in some defended animals (Robinson 1969).

Recently, however, researchers experimentally explored the switchable visual displays of some chemically defended prey (Kang et al. 2011; Umbers \& Mappes 2015; Kang et al. 2016) rising new questions and "rejuvenating" old ones in this field (Umbers et al. 2017).

\section{Costs and benefits of switchable aposematism.}

Physiological and/or anatomical mechanisms that allow signal switching might impose additional costs to the prey animals. Some of these costs might involve development and maintenance of such mechanism ("fixed" cost), while others might be paid every time the switching behavior occurs ("per-use" cost, terminologies adopted from Broom et al. (2010)).

On the other hand, a switchable signal can be beneficial in several ways. First, regardless of any aposematic function, a sudden switch may simply surprise the predator away (the "startling" effect (Ruxton et al. 2018)). The sudden switching might also accelerate the predator's learning about the aposematic prey (Kang et al. 2016). Finally, it might cut down unnecessary signal exposure because an animal can present the expensive signal only when needed (Grober 1988; De Cock \& Matthysen 1999; Umbers et al. 2017).

Among these benefits, the cost-reducing aspect is probably the most cumbersome to handle in empirical studies because the evolutionary cost of a signal is difficult to quantify or manipulate (Srygley 2004; Bohlin et al. 2008; Crothers et al. 2011; Lindstedt et al. 2016). 
121 Therefore, we chose to use a modeling approach to focus on the variety of costs of switchable

122 signals.

123

124

125

126

127

\section{Different types of costs related to switchable aposematism.}

In order to avoid confusion between the different types of costs involved in switchable

128 aposematism, we will use consistent specific expressions from now on. The energetic or material cost paid per every switching will now be called the 'switching cost.' The developmental and/or maintenance costs associated to the switching mechanism itself, which is always paid regardless how often the switching-signal is actually fired, will now be collectively called the 'switchability maintenance cost.' These two types of costs are expected to hinder the evolution of switchability. The signal-induced exposure to the predators will now be called the 'detectability cost.' All the other costs inherent to the active signal itself, e.g. energy consumption for producing sound or potential hindrance in foraging (Brandley et al. 2016) will now be called the 'signaling penalty.' These two types of costs are expected to hurdle the evolution of aposematism itself, but switchability may help relieve them. A generic term that encompasses and summarizes all of these negative effects will be the 'general cost(s) of signaling.' This general cost is expected to be optimized against the general benefit of signaling. Some of these terminologies will begin to appear in bold texts, indicating that the corresponding concept is directly modeled by a simulation variable.

We developed ApoSim, an individual-based simulation model to study this subject. In this model, computer-simulated predators and prey interact with each other, and their performances are under selection pressure. ApoSim is capable of exploring a very wide parameter space, but for this paper we assumed a special situation; the learning facilitation and startling effects of switchable signals are negligible. Under this assumption, we could explore the cost-reducing aspect of switchable aposematism in a clean, conservative scope. Users of the model software can easily conduct future studies in order to address questions regarding the startle effect or learning facilitation (see Supplementary Material).

155

\section{Previous knowledge pertaining to the general costs of switchable aposematism.}

Throughout the development of ApoSim, we had to consider a variety of relevant phenomena that have been suggested or reported. The sequential nature of post-attack switching that is dependent on the success of the pre-attack defensive measures (Broom et al. 2010; Ruxton et al. 2018), the 
161 baseline detectability of non-signaling prey (De Cock \& Matthysen 1999; Broom et al. 2010), the

162 fixed and per-use costs of switchability (Higginson \& Ruxton 2009; Broom et al. 2010), and

163 sensory and cognitive ability to detect predator's presence (Broom et al. 2010) have all been

164 under investigation. Notably, the interactions between these mechanisms and the general costs of

165 switchable aposematic signals is not fully understood.

166 The general cost of switchable signaling is not a single, readily measured value, but a

167 result of highly complex and relatively unexplored interactions. Energy consumption (Srygley

168 2004; Lindstedt et al. 2011), predator learning and behavior (Guilford 1994; Gamberale-Stille \&

169 Guilford 2004; Halpin \& Rowe 2017), the portion of the predators not deterred by the signal

170 (Williams et al. 2003; Mappes et al. 2014), the level of crypsis achievable when not signaling

171 (Bohlin et al. 2008; Willink et al. 2013; Umbers \& Mappes 2015), and many other aspects could

172 all affect the general cost of switchable signaling. Furthermore, some of these conditions can

173 change every time the signal is switched on. Due to this complexity, the study on switchable

174 aposematism is still in its beginning stage. Therefore, we carefully designed the simulation so

175 that it can embrace most of the above ideas in one framework.

176

177

178

179

180

181

182

183

184

185

186

187

188

189

190

191

192

193

194

195

196

197

198

199

200

\section{Scope of this study.}

Based on our simulation, we suggest new hypotheses regarding the evolution of switchable aposematism and test existing hypotheses in a context different from which they were originally formulated. First, we explore if variation in the selective penalty of the aposematic signal is sufficient to explain the observed diversity of switchable aposematism. Second, we determine how evolutionary success of different types of switchable aposematism is affected by increasing costs of switching. Third, we evaluate the existing hypotheses suggesting that moderate-range predator learning capabilities create the most favorable conditions for the evolution of aposematism (Speed 2001; Puurtinen \& Kaitala 2006). Fourth, we explore some previously proposed hypotheses about the effects of mixing of naïve and experienced predators on the evolution of various aposematic strategies (Endler \& Mappes 2004; Kang et al. 2011; Mappes et al. 2014; Kang et al. 2016). Fifth, we determine if and how the basal detectability of the prey affects evolution of aposematism. Finally, we explore several possible evolutionary pathways that can lead to switchable aposematism from non-switching and/or non-aposematic initial population.

Additionally, we provide our model and the source code so that the users can further explore the evolution of complete diversity of aposematic strategies. The model can simulate the interactions between these evolutionary components in a multidimensional evolutionary space. In addition, we provide a description of how to use this software to explore variables other than those used in this article. 


\section{Materials \& Methods}

203

204

\section{Core ideas and assumptions.}

205

206

\section{The prey behavioral strategy.}

207

We modeled the prey behavioral strategy as responses to three conditions: "resting," being "approached," and being "attacked." Specifically, a strategy is defined by three values of signal intensity (ranging from 0 to 1 ) each associated with one of the three conditions above. For example, a strategy could be written as the following: [(resting, 0.1), (approached, 0.1), (attacked, 0.7)]. Each number represents the signal intensity given in each condition. This strategy is a variant of post-attack switchable signaling, because the sudden increase in signal intensity (from 0.1 to 0.7 ) occurs once the predator attacks. Meanwhile, a permanently aposematic prey should have the same signal intensity for all three conditions. In the case of a pre-attack switcher, it should remain largely inconspicuous while resting, but switch to higher intensity when "approached." It should be noted that signal intensity is a one-dimensional value; we assumed that all signal varieties operate on the same common axis, and that the predators automatically generalize their knowledge about one signaling prey to all the others. Therefore, all prey signals are 'mimetic' to each other.

222

While signal intensity could technically take any value from 0 to 1 , we further simplified this variable for the sake of easier visualization. We assumed that the prey can only have three discrete levels of signals: "none" $(\mathrm{N})$, "low" (L), and "high" $(\mathrm{H})$ (the exact value of each level can be adjusted by the user). With these discrete levels, one could describe a behavioral strategy without using the numerical values for the signal intensity. For example, the pre-attack switchable aposematism could be written as [(resting, N), (approached, H), (attacked, H)].

We devised a three-letter annotation system as a shorthand for each behavioral strategy. The sequence of the three conditions is easy to remember as they are in the logical order of increasing threat level: resting, approached, and attacked. Therefore, the previous expression could be shortened to [NHH]. Likewise, one could easily understand that LLL is a permanent-

231 display strategy with low signal intensity (L). Similarly, NLH is a strategy of an animal that does not signal at rest $(\mathrm{N})$, responds to an approaching predator by switching to a weak pre-attack signal (L), and responds to a predator attack with a stronger signal (H). With this concise style, we could easily express complex ideas such as "competitive advantage of NLL over NLH" or "the change in LLL-NNH balance in the presence of NLLs" without repeating the lengthy explanations every time.

In summary, three signal intensity levels $(\mathrm{N}, \mathrm{L}$ and $\mathrm{H})$ are allowed in each of the three states (resting, approached and attacked), resulting in $3^{3}=27$ possible behavioral strategies. The user could configure whether a specific strategy can exist or not in the model. 


\section{The predator attack event.}

243

244 Given the basic structure of the prey behavioral strategies, it was clear that the predator should fired after an attack event, and it should affect the prey survival by influencing the chance of the subsequent attack(s).

initial attack event occurs after the prey's pre-attack behavior and before the post-attack behavior; a subsequent attack event occurs after the post-attack behavior. These two attack events act as junctions that naturally divide the whole interaction into three time steps: preinitial-attack period (step 1), inter-attack period (step 2), and the post-final-attack period (step 3).

\section{The scope of the simulated evolution.}

256

257

The model simulates competition for higher survival among different prey behavioral

258 strategies. In contrast, properties of the predator population are directly specified by the user. Hence, rather than simulating the predator-prey coevolution, the model focuses on the evolution of prey population given a certain fixed set of predator properties. With this approach, we could explore the direct effect of specific characteristics of the predatory guild.

\section{Overview of the model structure.}

We built the model with NetLogo 5.3.1, an agent-based modeling framework developed at Northwestern University (Wilensky 1999). The simulation code and graphical interface are published with the article. Note that the graphical interface was used only for testing, prototyping and pedagogical use; the exploration of the parameter space was programmatically executed by BehaviorSpace, a parameter-search tool bundled with NetLogo 5.3.1.

Our model, ApoSim, is capable of modeling startle and learning facilitation effects of switchable signals as well as a number of other ecological variables. However, here we describe only the mechanics relevant to our study questions. For the full description of the model variables, please refer to the Supplementary Materials and the source code.

The modeled world consists of a single species of 180 defended prey animals and a single species of 45 predators (Figure 1). Each individual prey occupies one grid square of the 2dimensional world (14 $\times 14$ grids). The prey distribution is globally random, and the model does not aim to simulate the kin-selection effect of gregariousness. Initially the prey population is a uniform mix of all possible behavioral strategies. 
280

281

282

283

284

285

286

287

288

289

290

291

292

293

294

295

296

297

298

299

300

301

302

303

304

305

306

307

308

309

310

311

312

313

314

315

316

317

318

The basic time unit of the simulation is the interaction frame (Table 1). 50 interaction frames forms one prey generation, after which the current prey individuals reproduce and die out. Each interaction frame includes three time steps. In each time step, every prey and predator individual follows specific set of steps coded in program's procedures. The names of the procedures, as they appear in the source code, are given in Table $\mathbf{1}$ and are detailed in the subsequent sections.

During each interaction frame, a predator could encounter and interact with an individual prey throughout the three time steps. During the interaction, the predator can detect the prey's repulsive taste, which reduces the predator motivation. The exposure to repulsive taste could lead to accumulation of the predator's aversive memory value from 0 to unlimited level. After enough interactions, the heightened level of aversive memory could cause the predator to refuse to attack this prey species. Also, the interaction could lower the survival chance of the prey, which begins as 1 and then subsequently decreases by multiplying by decimal modifiers. Actions of the predator and the prey determines the levels of the modifiers. Depending on how the interaction unfolds, the predator might fail to detect the prey or decide to abandon it. In such a case, the interaction is prematurely terminated, and the predator would idle for the rest of the interaction frame.

After 50 interaction frames (one prey generation) all prey enter the reproduction stage. First, the prey individuals die out according to the final values of survival chance. All the survivors have equal chance of reproduction. Reproduction is simulated by repeating the procedure of randomly choosing one of the survivors and generating one offspring of the same behavioral strategy until the carrying capacity (180 individuals) of the next generation is reached. After the entire reproduction stage is over, the survivors of the current generation dies immediately (non-overlapping generations). There is no simulation of offspring growth stages. There is no sexual reproduction, and each offspring exhibits the parental phenotype identically.

On the other hand, the predators do not have any fixed lifespan, and a preset number of fresh naïve predators randomly replace the old individuals according to the user-defined predator turnover rate at the end of each prey generation. We assumed that predators acquire the avoidance entirely through associative learning, not instinct. The predator learning speed is adjustable by the user. The predators are genetically homogeneous and not subject to natural selection.

\section{Detailed simulation structure.}

\section{Interaction frame: time step 1.}

"Predators-move" procedure. - At the beginning of each interaction frame, every predator moves to a random grid square which contains a prey. In our setup, the number of the predators 
319 is smaller than that of prey; this procedure leaves the majority of the prey without predator

320

321

322

323

324

325

326

327

328

329

330

331

332

333

334

335

336

337

338

339

340

341

342

343

344

345

346

347

348

349

350

351

352

353

354

355

356

357

encounter. Every prey is still in the "resting" stage.

"Prey-react" procedure. - Each prey detects the predator presence or absence in the grid square it is occupying, and it then switches or maintains its aposematic signal. After detecting the predator absence or presence, the prey then stays still or switches its signal intensity depending on its behavioral strategy. More specifically, each prey continues to be in "resting" state if no predator is in its grid square, and if one is present, then the prey attains the "approached" state instead. As detailed in the first section of Methods, the prey signal intensity is one of the three levels: none $(\mathrm{N})$, low $(\mathrm{L})$, or high $(\mathrm{H})$. Each level corresponds to a number ranging from 0 (minimum possible signal) to 1 (maximum possible signal), and the exact values could be set by the user.

In this study, we assumed that the prey had perfect sensory and cognitive ability to accurately determine the predator presence. We do recognize that ambush predators are major exceptions to this assumption, and therefore we do not intend to explain their ecology with our model.

"Prey-pay-cost" procedure. - Regardless whether it is encountering a predator or not, each prey has to pay the energetic and ecological price of its current behavior and appearance. Each prey is continuing its "resting" or "approached" state, and the cost-paying procedure runs like the following.

First, if the prey has switched the signal to a different level, such behavior might require extra energy and reduce its chance of survival. The user-adjusted variable switching cost (range 0 to 1 ) represents this effect, and the prey survival chance (range 0 to 1 ; initially at 1 ) decreases accordingly (Eq. 1). Note that we are using Roman numerals to mark the intermediate values used in sequential calculations (an example is "survival chance I" below). Also, we decided to use full-word names of the variables instead of single letter symbols because we think this will facilitate better and easier understanding of the whole model by the intended readership biologists who study aposematism.

Eq. 1 survival chance $I=$ current survival chance * $(1-$ switching cost $)$

Second, an animal with a switching mechanism needs to maintain it energetically and cope with the developmental complexity regardless whether the actual switching behavior has fired or not. In our model, a user-adjusted variable switchability maintenance cost (range 0 to 1 ) simulated this effect (Eq. 2).

Eq. 2 survival chance II = survival chance I * $(1-$ switchability maintenance cost $)$ 
358 Finally, an active state of the signal itself, be it switchable or permanent, might impose a variety

359 of burden of prey survival that are not explicitly modeled in our software. For instance, high

360 signal intensity could attract parasites that are not deterred by the unpalatability. If the prey's

361 mode of signaling involved sound generation or odor production, there would be extra energy

362 consumption. If the prey uses a physical device that can be shown, moved, or inflated, the prey

363 animal's mobility would suffer in addition to energy costs. A myriad of such effects are

364 summarized in our model as signaling penalty, a user-adjustable value from 0 to 1 . This is

365 weighted by signal intensity (Eq. 3).

366

367

368

369

370

371

372

373

374

375

376

377

378

Eq. 3 new survival chance $=$ survival chance II $*(1-$ signaling penalty $*$ signal intensity $)$

In principle, there would be a production and maintenance cost for all signals regardless switchability; some developmental burdens such as pigment production could be shared by permanent and switchable signals. We decided to exclude this variable from the model, as its effect is largely trivial and predictable - the costlier the signal maintenance, the lower the prevalence of signaling phenotypes.

"Predators-search" procedure. - Each predator investigates the grid square and detects the prey presence with a certain probability. This discovery chance (Eq. 4) is a function of basal detectability and signal intensity of the prey (which will all be in the "approached" state; only the prey sharing its grid square with a predator can be searched, so no "resting" prey is under consideration here). Basal detectability (range 0 to 1 ; user-adjusted variable) is a simplified representation of prey body shape, the habitat properties, the predator cognitive attributes, and

381

382 anything that can affect the minimum level of discovery chance. Hence, the discovery chance value is larger for higher basal detectability. It is additionally increased if the signal intensity

383

384 value is larger than 0 . The signal intensity effect is weighted by the maximally possible increase of the detectability ( 1 - basal detectability), so the final value is limited between 0 and 1 .

385 Unlike many other similar equations in the model, basal detectability and signal intensity have an additive, not a multiplicative, relationship. The rationale for this design is that both basal detectability and signal intensity should be able to contribute to predator discovery even if the other variable is close to zero or zero.

389

390

391

Eq. 4 discovery chance $=$ basal detectability + signal intensity $*(1$ - basal detectability $)$

"Predators-observe" procedure. - If a predator has detected a prey, then it observes the appearance of the target prey (which is still in its "approached" state). Conditional stimulus

394 (range 0 to 1) is a value that represented the intensity of all information that can mediate the

395

396 predator's associative learning regarding the prey. A high conditional stimulus value means that the prey has characteristics that allow easier memory buildup and recall. 
402

403

404

405

406

407

408

409

410

411

412

413

414

415

416

417

418

419

420

421

422

423

424

425

426

427

428

429

430

431

432

433

434

435

436

The information available to predator could have been come from either the prey's signal or its signal-independent inherent traits. Conditional stimulus is a function of prey signal intensity and basal cognitive cue (Eq. 5). Note that the formula is in the same format as Eq. 4; basal cognitive cue sets the baseline, and signal intensity determines the remaining portion so that the final value cannot exceed 1 .

Eq. 5 conditional stimulus $=$ basal cognitive cue + signal intensity $*(1-$ basal cognitive cue $)$

The prey body shape, the predator cognitive attributes, environmental conditions, and many other factors could govern the minimum level of associative learning and memory retrieval. Basal cognitive cue (range 0 to 1 ; user-adjusted variable) represents them. Note that basal detectability and basal cognitive cue are two different variables despite looking similar. Basal detectability affects the chance of discovery (Eq. 4), while basal cognitive cue is for the learning/recalling stimulus (Eq. 5). For example, a flying insect might be easily detectable (high basal detectability) but it could be difficult to learn or recall from the appearance, because it looks too similar to many other profitable insects (low basal cognitive cue).

"Predators-analyze" procedure. - From the information observed from the prey, the predator attempts to determine its attractiveness based on memory as well as taste (if available). After perceiving a conditional stimulus, each predator assesses prey attractiveness. Prey attractiveness (range 0 to 1 ) is a function of learned repulsiveness and instinctive repulsiveness, both with a range from 0 to 1 (Eq. 6). For a prey to be highly attractive, both learned and instinctive repulsiveness should be close to 0 .

Eq. 6 prey attractiveness $=(1$ - learned repulsiveness $) *(1-$ instinctive repulsiveness $)$

The predator's impression from the prey is determined by the level of memory, the strength of cues that recall the memory, and the currently felt taste (if available). Learned repulsiveness is determined by the level of the conditional stimulus (Eq. 5) and the aversive memory. The learned repulsiveness is forced to be within the range of $[0,1]$, as seen in Eq. 7. Instinctive repulsiveness is solely determined by the unconditional stimulus (range 0 to 1 ) the predator is currently feeling (Eq. 8). Both unconditional stimulus and conditional stimulus are terminologies following the convention in classical conditioning (Sweatt 2009); they themselves are not 'memory,' but an association between them (aversive memory) is. Also, the model software can be configured to include the 'startle' effect in the instinctive repulsiveness calculation; see Supplementary Materials.

Eq. 7 learned repulsiveness $=$ minimum $\left\{\begin{array}{c}1 \\ \text { aversive memory } * \text { conditional stimulus }\end{array}\right.$

Eq. 8 instinctive repulsiveness $=$ unconditional stimulus 
438 The predator's past memory and the cues that recall it are both necessary for a learned avoidance

439

440

441

442

443

444

445

446

447

448

449

450

451

452

453

454

455

456

457

458

459

460

461

462

463

464

465

466

467

468

469

470

471

472

473

474

475

476 to take effect. Unlike all the other values that range from 0 to 1 , aversive memory could be any non-negative number and had no upper bound. Therefore, sufficiently high aversive memory could bring the learned repulsiveness up to 1 even if the conditional stimulus (Eq. 5) is very small. On the other hand, a completely naïve predator with no aversive memory, will have no learned repulsiveness regardless of the conditional stimulus. The mechanics of the aversive memory buildup will be detailed in the subsequent sections (Eq. 12a, b).

The 'taste' information is only accessible after the predator made at least one attack event. The unconditional stimulus represented all modes of unprofitability that can be sensed by the predator only during attack events. In the current time step 1, the predator has not yet tasted the prey (which is still in its "approached" state), so the received unconditional stimulus is 0 (Eq 8.9). In the later time steps, there will be taste information available, and the mechanics will be detailed in the subsequent sections.

Eq. 8.9 unconditional stimulus $=0$

"Predators-decide" procedure. - From the predator's impression of the target prey (which is still in the "approached" state), the "decision" to attack (or not) is made. Motivation (range 0 to 1) began from 1 whenever the predator encountered a new prey. After assessing the prey attractiveness (Eq. 6), each predator modifies its motivation according to it. In order to maintain high motivation, the prey attractiveness should remain close to 1 (Eq. 9).

Eq. 9 new motivation $=$ current motivation $*$ prey attractiveness

This value ("new motivation"), with range 0 to 1 , is the probability of deciding to attack the focal prey. After the "decision" is determined, it is internally stored until the actual action is conducted in the next time step.

\section{Interaction frame: time step 2.}

"Predators-initial-act" procedure. - Each predator conducts an action (attack or abandonment) according to the "decision" made in the previous step. The probability of attack was the value of "new motivation" (Eq. 9) at the end of the time step 1. If the predator decided not to attack, then it abandons the prey (which will revert to "resting" from the hitherto "approached" state) and will remain idle for the rest of the interaction frame. If the predator decided to attack, the predator-prey pair undergoes an initial attack event. In such an event, the prey (now in "attacked" instead of hitherto "approached" state) suffers a decrement in its survival chance 
477 (range 0 to 1 ). This effect is governed by the user-adjusted variable initial attack damage (range 4780 to 1; Eq. 10).

479

Eq. 10 new survival chance $=$ current survival chance $*(1-$ initial attack damage $)$

"Predators-feel" procedure. - After attacking a prey (which is now in "attacked" state), each predator receives taste information from handling the prey. Therefore, the predator could now update the unconditional stimulus value. In the current time step 2, the predator is performing its initial attack, so the information is updated from 0 to the repulsive taste (range 0 to 1 ; useradjusted variable) of the prey (Eq. 11). We assumed that the prey repulsive taste is a homogeneous characteristic across all prey, and there was no mutation or variation modeled. See Discussion for the implications of this assumption.

\section{Eq. 11 unconditional stimulus $=$ repulsive taste}

"Prey-react" procedure. - As a result of being attacked or abandoned by a predator, each prey is already in the "attacked" or "resting" state, and it reacts to that situation. The details of signal intensity change are identical to the "prey-react" procedure of the time step 1. In other words, based on its behavioral strategy (as described in the first section of Methods), the prey changes its signal intensity or remained in the current appearance.

\section{"Prey-pay-cost" procedure. - Each prey (either in its "resting" or "attacked" state) underwent a} series of survival chance decrements, following the principles identical to the time step 1 (Eq. $1,2,3)$. Especially, if the reaction involved switching of signal intensity to a different level, the prey paid the switching cost (Eq. 1).

"Predators-observe" procedure. - As it did in the previous time step 1, each predator experienced the conditional stimulus (Eq. 5) again, but this time calculated with the current value of signal intensity displayed in step 2 (after predator initial attack - the "attacked" state of the prey).

"Predators-learn" procedure. The predator learns the association between the prey and the negative taste, and the speed of learning is determined by how striking the prey's appearance (in its "attacked" state) is, how unpleasant the taste is, and how the program user set the global adjustment to all learning process. Technically speaking, each predator builds up aversive memory (Eq. 12a, b) based on the newly updated conditional stimulus (Eq. 5), unconditional stimulus (see the "predators-feel" procedure above), and a user-adjusted coefficient called

515 learning speed.

Eq. 12a new aversive memory $=$ current aversive memory + aversive memory increment 
518 Eq. 12b aversive memory increment $=$ learning speed $*$ repulsive taste $*$ conditional stimulus

519

520

Aversive memory is any non-negative value with no upper bound, as explained in Eq. 7 and the

521 accompanying text.

522

For the sake of simplicity, there was no memory decay modeled, and the predator

523

524 population turnover was the only source of collective memory decrement. We made this assumption because taste aversions tend to have very high retention time (Steinert et al. 1980;

525 Elkins 1984). It is possible for the users to modify the code and include memory decay if 526 desired.

We allowed the speed of learning to be adjusted over very wide range. Similar to

529 aversive memory, the user-adjustable learning speed also has no upper bound; very fast learning could induce substantial build-up of aversive memory even when both unconditional stimulus and conditional stimulus are fairly weak. For perspective, we used learning speed values from 0 to 1000 in our study. Learning speed is an abstraction of the predator sensory and cognitive abilities as well as the environmental difficulties that might hinder acquisition of the aversion. For example, if Batesian mimics or other harmless yet similar-looking food sources are present in the habitat, the predator aversion learning would require longer time. The user might want to change the learning speed setting to form hypotheses involving such effects.

536

"Predators-analyze" procedure. - Each predator uses the updated aversive memory, conditional stimulus, unconditional stimulus values to determine the prey attractiveness (Eq. 6) again. The prey is still in its "attacked" state.

540

"Predators-decide" procedure. - Each predator updates its motivation (Eq. 9) based on the reassessed prey attractiveness. The new motivation value is the probability to attack the prey in the next time step 3. The prey is still in the "attacked" state.

\section{Interaction frame: time step 3.}

"Predators-subsequent-act" procedure. It is almost identical to the "predators-initial-act" procedure in the previous time step. This time its action is either a subsequent attack event (prey stays in the "attacked" state) or an abandonment (prey reverts to "resting" state), and the prey survival chance is affected by subsequent attack damage (range 0 to 1 ), another useradjusted variable (Eq. 13).

553

Eq. 13 new survival chance $=$ current survival chance $*(1-$ subsequent attack damage $)$ 
556 "Predators-feel" procedure. Each attacking predator receives the updated unconditional

557 stimulus as it did in the previous time step. Since this is a subsequent attack, the information

558 would not be different from what it felt in the initial attack. Each prey is either in "attacked" or

559 "resting" state.

560

561 "Prey-react" procedure. - Each prey reacts to the subsequent attack in the same manner it did in

562 the previous time step 1 and 2 , following its behavioral strategy (see the first section of

563 Methods). Depending on whether the subsequent attack was executed, the prey is currently in

564 either "attacked" or "resting" state.

565 Note that the predator is going to leave the site afterward regardless of the prey response,

566 as the simulation will not give any more subsequent attack event. Therefore, in some situations,

567 the prey would gain little benefit from reacting to this last attack from the predator. However, we

568 assumed that the prey cannot know if the predator interaction is coming to an end, and that it will

569 unconditionally display the signal as long as its behavioral strategy assigns a response to attack.

570 In other situations, strongly reacting until the end of the attack (and thus giving prolonged

571 stimulus to help predator learning) may increase prey fitness, if the prey or its offspring is likely

572 to encounter that identical predator individual again in the future.

573

574 "Prey-pay-cost" procedure. - Each prey (in either "attacked" or "resting" state) suffers a drop in

575 survival chance, as it did in the previous time step 1 and 2 (Eq. 1, 2, 3).

576

577 "Predators-observe" procedure. - Each predator gathers the conditional stimulus (Eq. 5) based

578 on the updated signal intensity information (from the prey in "attacked" state), as it did in the

579 previous time step 1 and 2.

580

581

582

583

584

"Predators-learn" procedure. - Each predator increments its aversive memory (Eq. 12a, b) with the updated conditional (Eq. 5) and unconditional (see the "predators-feel" procedure) stimuli as well as the user-adjusted learning speed. Each prey is still in either "attacked" or "resting" state.

585

586

587

588

589

\section{Reproduction stage.}

590

591

592

"Prey-selectively-die" procedure. - Each prey survives or dies according to the final value of its survival chance.

593 
594 "Prey-lay" procedure. - One of the surviving prey individuals is randomly chosen to generate a 595 clone that will live in the next generation. The process is repeated until the carrying capacity 596 (180 individuals in our case) of the next generation is all filled.

597

598

599

600

601

602

603

604

605

606

607

608

609

610

611

612

613

614

615

616

617

618

619

620

621

622

623

624

625

626

627

628

629

630

631

632

633

"All-prey-die" procedure. - Every prey of the current generation dies out, only leaving the nextgeneration population.

"Prevent-extinction" procedure. - In our model, a given prey phenotype could not undergo a complete extinction. As a result of the prior procedures, a prey behavioral strategy could have temporarily gone to extinction at the previous procedure. Then, the program forcefully rescues the situation by generating a new prey individual with the extinct trait at the expense of a random existing individual. It was a design choice to prevent premature fixation and improve the robustness of the model outcome. With this procedure, the model could maintain the variation without any mutation or recombination; it helped us to minimize quantitative assumptions that are not directly related to our question.

"Predators-turnover" procedure. - A number of naïve predators enter the world and replace some of the old predators. The user-adjusted variable predator turnover rate determines the proportion of the predators that are replaced by fresh ones, and the turnover occurs at the onset of the next prey generation. The new predators joining the model have zero aversive memory.

\section{Final outcome: the winning strategy.}

After 500 prey generations we determined the outcome of the simulation. The winning strategy was defined as the most abundant behavioral strategy among prey.

\section{The settings used for this article.}

ApoSim has many user-adjusted settings and variables (Table 2), and it was impractical to explore all the possible combinations in one focused study. Instead, we focused here on a subset of questions arising from the existing literature as outlined in the Introduction. We asked how several types of costs that prey incurs affect the evolutionary outcome, and how does the outcome depend on the learning speed and the influx of naïve predators into the system.

We decided to vary only five variables, as five-dimensional data would be close to the limit of meaningful visualization. We chose three variables relevant to general costs of signaling: basal detectability, signaling penalty, and penalty of switchable signals (which was composed of switching cost and switchability maintenance cost). We used two independent variables representing the properties of the predators: predator turnover and learning speed. Note that 
634 technically we varied 6 variables, but switching cost and switchability maintenance cost were

635 similar in nature, and we decided to co-vary them assuming positive correlation (which may not

636 be true in some natural systems).

637 The combination of these five independent variables led to 29376 different conditions,

638 and we repeated the runs three times in each condition. This resulted in 88128 outcomes in total.

639 Besides these five independent variables, we treated every other user-adjustable variable as a

640 controlled variable; we gave a fixed, reasonable value for each of them (Table 2). We want to

641 emphasize that a future user of this software can choose completely different sets of independent

642 variables.

643 Due to this incomplete search of the parameter space, our model result should be

644 interpreted with caution. Our choice of control variables (Table 2) is supported by parameter-

645 bracketing results (Supplementary Figure 3); however, it is still a set of arbitrary assumptions

646 that should not be directly related to quantitative measurements from the real world.

647 Among the variables shown in Table 2, the list of behavioral strategies needs more

648 clarification. As we mentioned in the first section of Methods, one of our core ideas is that the

649 user can describe a variety of behavioral strategies in the three-letter notation.

650 Out of 27 possible combinations, we chose 9 strategies for this study based on the following 651 assumptions. First, the prey can have only one switching opportunity at maximum. Therefore,

652 the strategy can be either one of the pre-attack or post-attack switching, but not both. This was to

653 aid visualization by making the competitive alternatives highly contrasting; compromised

654 intermediates can be difficult to plot and describe. Second, we assumed that increasing threat

655 level can only be associated to increasing levels of signal intensity; the prey should stay at the

656 current level or switch to a higher signal when freshly "approached" or "attacked." In the current

657 version of the software, the predator detection check is only done once. This limitation made it

658 useless to hide after initial interaction, because such behavior cannot alter the outcome of that

659 encounter. With these two restraints, the possible combination of strategies reduced to 9 as seen

660 in Table 2.

661

662

663

664

Results

665

666 Two-dimensional mini-plots (Figure 2) are combined in three-dimensional meta-plots (Figure

667 3) that contain the outcomes of 88128 model runs. Here, we briefly describe the resulting

668 Figure 3, highlighting comparisons among the figure elements that later in Discussion will be

669 invoked again. From now on, we will regard Figure 3B, for the moderate switching cost and

670 switchability maintenance cost, as the standard result analyzed in detail. We will then describe

671 differences in Figure 3A or 3C compared to Figure 3B.

672

673

In Figure 3B, NNH, NHH, or HHH behavioral strategies generally appeared in the moderate learning speed range. Conditions with extremely slow-learning predators favored 
674 NNN, the none-signal strategy (e.g. "a" of Figure 3B). As the learning speed increased, 675 strategies with high signal intensity began to appear (e.g. the transition from "a" to "b" of

676 Figure 3B). The opposite extreme condition with fast-learning predators also favored NNN (e.g.

677 "d" of Figure 3B). There was a tendency of smooth tapering from high- to low- and finally to

678 no-signal strategies as the learning speed increased (e.g. the transition from "e" and "f" columns

679 to "d" column in Figure 3B).

680 Conditions with higher basal detectability and higher learning speed selected the pre-

681 attack or permanent signaling strategies, while lower basal detectability and lower learning

682 speed favored post-attack strategies (e.g. contrasts between "b," "ce" and " $f$ " in Figure 3B). If the

683 basal detectability was extremely high while the predator learning speed was extremely slow,

684 then there was no stable winner, and the outcome was mostly random (e.g. "g" of Figure 3B).

685

686

687

688

If learning speed and basal detectability were both in the moderate range, the signaling penalty dominated the performance of behavioral strategies. The lower signaling penalty led to stabilization of permanent signals; higher signaling penalty favored post-attack signals; preattack signals won in the middle ground (e.g. "h" of Figure 3B).

689

Finally, if the predator turnover was high, the influence on the outcome appeared

690 mostly identical to the slowing down of the effective learning speed. For example, in Figure 3B, the shift from "e" to "b" (decrease in learning speed) was similar to the shift from "e" to " $\mathbf{p}$ " (increase in predator turnover.) However, when the learning speed was high, the predator

693 turnover rate was a determining factor for the emergence of the post-attack signals: if the turnover was low, higher learning speed conditions mostly selected permanent or no signals strategies (e.g. "k" of Figure 3B); if the turnover was high, post-attack signals were also selected (e.g. "l" of Figure 3B).

697

698

Figure 3A shows the corresponding set of results with no fixed or per-use costs in

699

700

701

702

703

704

705

706

707

708

709

710

711

712 switching signals (i.e. switching cost $=0$ and switchability maintenance cost $=0$; Table 2 ). In this condition, permanent signal strategies disappeared almost entirely, and the switching strategies replaced them. Another difference is that the post-attack switching strategies became more common in normally unfavorable, extreme conditions.

Figure 3C shows results for situation in which the switching phenotypes pay very high costs (i.e. switching cost $=0.016$ and switchability maintenance cost $=0.000255$; Table 2 ). The figure shows that the no-signal strategies replaced most of the switching strategies. The switching phenotypes survived only in the narrow zone of moderate learning speed, high basal detectability, and extremely high signaling penalty.

To assess the robustness of the result, we have run simulations equivalent to Figure 3 with different levels of control variables (Supplementary Figure 3). Readers should be aware that however reasonable they are, our choices of control variables and their values are strictly arbitrary. Furthermore, as apparent in the Methods, the model structure is an abstract and simplified simulation, not a faithful reconstruction of a complex natural system. Therefore, the numerical values form the model should be interpreted as generic tendencies, rather than exact 
713 quantitative predictions of relationships that could be empirically discoverable in the real natural

714 systems.

715

716

717

718

Discussion

719

720

721

The results of the model (Figure 3) help our understanding of possible evolutionary transitions

722 between various forms of aposematic adaptations in prey. For instance, a defended prey may undergo evolutionary transitions from NNN (non-signaling) to $\mathrm{NHH}$ or $\mathrm{NNH}$ (examples of

723 switchable aposematic signals), from NNN to LLL or HHH (permanent aposematism), or from

724 $\mathrm{HHH}$ to NNH or NNN. Our model visualizes how such evolutionary transitions may be driven

725 by changes in ecology of predators and prey. Here, we will first discuss in details the conditions

726 that favor specific anti-predatory adaptations by focusing separately on each of the five

727 independent variables (Table 3). Then we will discuss possible evolutionary scenarios towards

728 switchable aposematic signals. Finally, we will also look closer at some of the existing and novel

729 hypotheses about the evolution of various forms of aposematism and how the model may

730 contribute to understanding of their evolution. To achieve clarity and precision of our

731

732 presentation, here we refer by number to relevant figures and tables in the Results.

733

734

735

736

737

738

739

740

741

742

743

744

745

746

747

\section{Predator learning and population turnover.}

The results are consistent with the existing theoretical coverage of the effects of predator learning and forgetting on prey aposematic signals (Speed 2001). For example, some of our model runs included predators whose learning speed is so extremely slow that the aposematic signals become useless. This condition is seen in "a" of Figure 3B. Increase in the predator learning speed eventually leads to the relatively abrupt appearance of the strongest possible signals $(\mathrm{H})$, be it switchable (Figure 3A, B) or not (Figure 3C; here the permanent signals are less costly). One such pattern is visible in the transitions from "a" to "b" in Figure 3B. This suggests that there may be a threshold of predator learning speed, below which aposematism (switchable or permanent) cannot outcompete the cryptic forms. This is because the prey cannot give infinitely effective signal while the predator learning speed can reach near zero; below some point of learning speed the prey should fall back to crypticity instead of investing more into the signal. When the predator learning speed is barely above this crypticity-aposematism

748 threshold, strong signals (H) are observed (e.g. "b" or "c" of Figure 3B) because high signal

749 strength is needed to cause efficient learning in the relatively slow learning predators.

750

751

As seen in Figure 3B, the crypticity-aposematism threshold is highly dependent on the predator turnover and basal detectability. Predator turnover determines the benefit of 
752 aposematism, as it determines the residence time of the educated predators; basal detectability,

753 the baseline chance of being discovered, determines the benefit of crypticity.

754 Slightly above the crypticity-aposematism threshold, the post-attack aposematism frequently

755 dominated the runs (e.g. from "a" to "b" in Figure 3B). When predators learn slowly there is a

756 higher chance of encountering a predator with little knowledge, and it is more likely to be

757 attacked upon discovery. Under this condition, post-attack strategies, which minimize the risk of

758 detection, should be favored if switching-related costs are not too high (Figure 3A, B).

759 On the other extreme of learning speed, our model shows an alternative situation. Under

760 very high learning speed (e.g. "d" of Figure 3B), NNN strategies are the most successful ones.

761 In our model, predators can learn from the prey basal cognitive cue even when no signal is

762 given from the prey. If learning speed is high enough, this basal learning can grant adequate

763 protection. Hence, in this situation the basal cognitive cue fulfils aposematic function; it should

764 be noted that the phenotype NNN is actually protected by aversive learning unlike the situation

765 at the slower extreme of learning speed. It is possible that some fast-learning predators may

766 indeed not need any auxiliary signal from the prey in order to recognize it as defended. For

767 instance, it has been demonstrated that oriental tits can learn the basic, inconspicuous

768 morphological features of an insect as cues of unprofitability, albeit at a slower rate compared to

769 learning of a typical aposematic signal (Kang et al. 2016). This signal-free, cue-based

770 aposematism is expected to have a widespread significance in evolution of many predator-prey

771 systems, because there exist a number of cue-based protective mimicry (de Solan \& Aubier

772 2019).

773 The transition toward this 'inconspicuous' form of aposematism appears to be more

774 gradual (e.g. from "m" to "d" in Figure 3B) compared to the relatively abrupt transition between

775 NNN and signaling strategies (e.g. from "a" to "b" in Figure 3B) at the slower learning speeds,

776 which we have already discussed. This effect of a very high learning rate could be inferred from

777 earlier models (Speed 2001; Puurtinen \& Kaitala 2006). Especially, the gradual transition toward

778 weaker conspicuousness fits well to the situation described in seasonal predator psychology

779 studies (Endler \& Mappes 2004; Mappes et al. 2014).

780 The effect of the decreased predator population turnover largely corresponds to the

781 effect of increased learning speed. As a result, the entire 3-dimensional plot is

782 compressed/shifted to the right side (requiring higher learning speed) as predator turnover

783 increases. This is because the influx of naive predators hinders the collective memory build-up in

784 the predator population, and the average predator behavior for a given prey becomes similar to

785 what can be expected in slower-learning but lower-turnover predator population.

786 However, the high predator turnover leads to a unique outcome with the very fast-

787 learning predators that can associate prey defense with the prey basal cognitive cue. As apparent

788 in "l" of Figure 3B, which can be contrasted to " $k$ " of the same figure, high predator turnover

789 favors the evolution of weak post-attack aposematism when the predator learning speed is

790 sufficiently fast. In this scenario, the predator knowledge level is highly variable (because of the

791 high turnover), and the prey cannot know if the approaching predator is a naive or experienced 
792

793

794

795

796

797

798

799

800

801

802

803

804

805

806

807

808

809

810

811

812

813

814

815

816

817

818

819

820

821

822

823

824

825

826

827

828

829

830

831

832

one. Therefore, the post-attack signal, which would be useless if the predator population was full of well-educated predators, is still needed to educate and deter the fresher portion of the predator population.

The mix of experienced and naïve predators has been a popular topic in studies of permanent aposematism (Lindstrom et al. 1999; Speed 2001; Svadova et al. 2009). Especially, the studies on the seasonal variation of predator knowledge (Endler \& Mappes 2004; Mappes et al. 2014) provide highly advanced analyses about the continuous influx of naïve predators and the need to repeatedly educate them. It was suggested that the post-attack display of the spotted lanternfly, Lycorma delicatula, is an adaptation to this circumstance (Kang et al. 2011; Kang et al. 2016). The results of our simulations indeed confirm that the weak post-attack switchable aposematism is actually the best strategy to deal with the mix of naïve and experienced predators of high learning speed.

\section{Basal detectability.}

Empirical and theoretical studies about deimatism (startle display) and post-attack defense have already discussed the general effect of the basal detectability (Broom et al. 2010; Willink et al. 2013; Umbers et al. 2015). However, to our knowledge, there is no currently available literature that focuses on evolution of switchable aposematism while overviewing the importance of the basal detectability along with other driving forces.

Our results are consistent with the general prediction that low basal detectability would favor the post-attack aposematism (e.g. panel "b" in Figure 3B) rather than the pre-attack or the permanent aposematic signals (e.g. panel "c" in Figure 3B). Initiation of an attack by a predator is a clear indication that the prey has been spotted. But before that moment of attack there is always a chance that the approaching predator has not detected the prey, especially for prey with low basal detectability. In this situation, it would not be beneficial to the prey to use a preattack display because such a behavior would reveal its presence to the predator, who otherwise is unlikely to be aware of its location. Similarly, the prey with permanent aposematic signals would entirely lose the opportunity to benefit from the potential crypticity. On the other hand, the post-attack signals are only emitted when the predator has already detected the prey and decided to attack (Broom et al. 2010). In such a scenario, giving post-attack aposematic signal cannot increase the risk of detection, as it is already detected. Hence, if the prey can energetically and ecologically afford the brief display of the signal, the post-attack switch-on can only help the prey, not harm it (unless the predator is more intrigued by the signal received during the attack). Therefore, post-attack signals can be a viable strategy in the conditions that are not favorable to pre-attack or permanent alternatives.

Higher basal detectability, on the other hand, effectively lowers the opportunity cost of aposematism. As an approaching predator is likely to detect the conspicuous prey anyway, the pre-attack or permanent aposematism would not bring much additional risk. As discussed before, the low learning speed is a condition where post-attack signals generally prevail. However, the 
833 high basal detectability and the reduced opportunity cost can bring the balance toward pre-

834 attack/permanent aposematism (transition from "b" to "c" of Figure 3B). If the additional risk

835 is negligible, pre-attack or permanent aposematism offer faster memory retrieval which grants

836 the prey further protection.

837 If the predator learning speed is too low in this condition, then the signal is void of both 838 risk and benefit - it may not significantly add to either detection risk or predator deterrence. In

839 this situation, the selection pressure on aposematism-crypticity axis is largely absent, and no 840 specific strategy would be clearly favored (e.g. "g" of Figure 3B) under our set of assumptions.

841

842

843

844

845

846

847

848

849

850

851

852

853

854

855

856

857

858

859

860

861

862

863

864

865

866

867

868

869

870

871

872

\section{Costs of signals.}

The general effect of the various types of costs on the evolution of post and pre- attack defenses have been analyzed earlier (Broom et al. 2010). However, to our knowledge, there had been no overarching theoretical perspective that shows the full implications of these costs when faced with learning-capable predator population. Our results provide a solid background to discuss the interactions between costs of a signal and costs of its switchability pertaining to the evolution of aposematism.

The signaling penalty is a value that represents the energetic cost of the signal as well as the exposure to the potentially signal-unfriendly environment (Table 3). If other variables have moderate values, the increase in signaling penalty leads from permanent to pre-attack, and to post-attack aposematism (as seen in "h" of Figure 3B and other similar mini-plots). As the signal is given for shorter and shorter duration along the above sequence, it is the logical order to constrain the increasing signaling penalty cost into an affordable range. However, as seen in most of the other mini-plots in Figure 3B, one should note that if other variables do not favor certain forms of aposematism, the signaling penalty alone - even when extremely high or low is insufficient to promote all possible types of aposematism.

Figure 3A shows the model outcomes when switching cost and switchability maintenance cost (together representing the "penalty of switchable signals") are absent while signaling penalty is present. In this condition, the permanent signals would be generally inferior to the switchable alternatives because of the longer duration of unnecessary exposure. Without any additional cost, the switchability provides finer control over the signal intensity fitting the circumstances. Therefore, the permanent aposematism present in Figure 3B mostly disappeared in Figure 3A.

Though less pronounced than in the permanent signals, some difference in the post-attack signal is also visible in Figure 3A; the post-attack signals are more likely to stabilize in signalunfriendly extremes. Being switchable, post-attack signals have two types of costs - the signaling penalty (cost associated to the signal intensity) and the switching-related costs (the fixed switchability maintenance cost and the per-use switching cost). Considering that the post-attack signal is presented only after a predator attack, the signaling penalty would be 
873 largely avoidable during the most of prey lifetime. Therefore, the reduction of switching-related

874 cost could substantially alter the cost-benefit balance of this strategy, which can be seen from the

875 difference between Figure 3A and 3B.

876 Figure 3C shows the other end of the spectrum. It depicts the model outcome when the

877 signal-switching behavior and the maintenance of switchability are very costly. Naturally, almost

878 all switchable signals disappeared, and permanent aposematism became more common. In

879 comparison to Figure 3B, one can see that the pre-attack signals mostly changed to the

880 permanent signals while post-attack signals generally reverted to permanent none-color strategy.

881 Permanent signal is a good substitute for pre-attack signal except for the lengthened display

882 duration; in predator deterrence, they serve essentially the same purpose. On the other hand, the

883 stability of the post-attack aposematism is mostly due to the utilization of crypticity while not

884 being attacked. Therefore, if switchable signals are not an option due to high costs, the post-

885 attack strategy is replaced with the no-signal strategy that maintains the benefits of crypticity.

886

887

888

889

890

891

892

893

894

895

896

897

898

899

900

901

902

903

904

905

906

907

908

\section{Conditions for evolution of switchable signals.}

Based on the model results, we hypothesize that the relative values of the two types of cost, the signaling penalty and the penalty of switching, predict the evolution of switchable or permanent signals (Figure 4). As the costs of switchable signals increase from Figure 3A, 3B to 3C, the switchability-favoring range of signaling penalty narrows down (e.g. from the whole range in panel "o" in Figure 3A to none in in panel "p" o, we deliberately configured the software to exclude any startling ('deimatic') or learning facilitation effects of the switched signals. In the setup used in this paper, the strength of the signal being switched on is the only relevant factor in predator psychology; the fact that it was switched does not have any effect except that it imposes some cost to the prey. This decision helped us to study the cost-saving aspects of switchable aposematism without further complications. However, this assumption is far from reality (Umbers et al. 2015; Kang et al. 2016; Ruxton et al. 2018), and the model predicts that the switchable aposematism would be more favored if startle and facilitation effects could provide survival advantages (Supplementary Figure 1).

909

910

\section{Visualizing hypothetical evolutionary pathways leading to aposematism.}

Although our model is not designed to directly imitate predator-prey coevolution, its results (such as Figure 3) can provide a useful aid to contemplate the evolutionary effect of changes in environment and in predator ecological guild. Figure 5 shows how such changes may mediate a variety of possible evolutionary pathways to different forms of aposematism in the defended

911 (unprofitable) prey.

912 
913 Scenario "a": highly detectable prey invokes learning in predators - The path "a" of Figure 5

914 represents a hypothetical scenario starting from non-cryptic and non-aposematic prey

915 phenotypes. The ancestral non-crypticity (high basal detectability) could have emerged by

916 chance, or due to selective processes other than aposematic signaling. Examples of such

917 conditions include sexual selection and conspicuous behaviors such as flight. Our model predicts

918 that such traits would affect the existing predation pressure very little if basal detectability is

919 high and the learning speed is slow (see Basal Detectability section of Discussion). Once the

920 defended prey has acquired those non-cryptic unique characteristics that are different from the

921 undefended ones in the same ecosystem, a selection pressure to distinguish them may apply to

922 the predators. This can lead to an increase in predator learning speed, which may in turn favor

923 signaling strategies in prey, thereby initiating a true aposematic interaction.

924 This scenario may be considered in sexual selection context (Maan \& Cummings 2009; Crothers

925 et al. 2011; Ruxton et al. 2018), because it provides a straightforward condition that can lead to

926 highly conspicuous appearances before aposematic evolution occurs. Another important example

927 regarding this pathway is the mobility benefit (Speed et al. 2010). If the prey is already

928 conspicuously mobile due to the need in resource collection, this is a condition that highly favors

929 the evolution of aposematism. Our visualization in "a" of Figure $\mathbf{5}$ is consistent with those two

930 scenarios and expands them with the insights gained from other related variables (Figure 3).

931

932 Scenario "b": quick-learning predators may provide a starting ground for evolution of

933 aposematism - Path "b" of Figure 5 illustrates a hypothesis that the ancestral predators have very

934 high learning capability. In this scenario, the basal cognitive cue - the minimal uniqueness in

935 body shape and behavior that arose from prey ecology - has been already performing an

936 antipredatory aposematic function (see Predator learning and population turnover above).

937 However, the variation in predator intelligence could have demanded some slight 'nudge' to help

938 the defended prey in being correctly discriminated. Once the prey evolves this minimal cognitive

939 aid that increases its survival, then this frees up the predators from the burden of cognitive and

940 behavioral investment that enabled the initial identification. This degradation of learning

941 capabilities then could have begun the down-spiral toward highly conspicuous aposematic

942 systems. In the final stages, as in the previous scenario, the relative ratio between signaling and

943 switching penalty determines whether permanent or switchable aposematism is established.

944 Alternatively, the path "b" is also consistent with a scenario that does not assume co-evolution

945 but simply represents a change of ecological guild of predators to which the prey is exposed:

946 from extremely fast learners to intermediately fast learning predators. One can easily imagine

947 that a prey invading new habitats may occasionally experience such changes. Similarly, a sudden

948 or gradual change of predators' guild composition may shift the prey conditions from facing the

949 "very fast learning" predators to facing "moderately fast learning" predators. By considering

950 such ecologically driven gradual evolution of prey signals, the scenarios along pathway "b"

951 directly provide alternative solutions to the controversy surrounding the possibility of gradual 
952 development of aposematism through co-evolution (Lindstrom et al. 1999; Endler \& Mappes

953 2004; Ruxton et al. 2018).

954

955

Scenario "c": post-attack switchable aposematism as ancestral form of aposematism - It is

956 reasonable that a prey at the post-attack stage would be more easily identified than at the pre-

957 attack stage. After being attacked the prey would attempt to struggle or flee, revealing the prey's

958 various body parts, behavioral peculiarities, and other information. Even if the predators have not

959 used this information yet, the prey might have been already under selection to intensify this post-

960 attack behaviors in order to increase survival through escape. This may lead to more and more

961 unique and conspicuous prey reactions, not because the conspicuousness was needed but as a byproduct of selection toward effective escape behaviors. It is feasible to assume that this intensification of post-attack behavior involves increase in its memorability to the predator. The higher memorability can effectively increase the learning speed, shifting the conditions from favoring "none-signal" (gray shaded panel in Figure 5) to those favoring post-attack signal (blue panels). Alternatively, the predators might evolve better cognitive ability (learning speed) to recognize the post-attack behavior of the defended prey because it may increase their foraging efficiency. Either way or both, our model predicts that the shift in the learning speed would lead to a new selection regime that will drive strong post-attack aposematism.

970 An alternative version of the above process may involve a shift from ecological conditions favoring no signaling (gray panels in Figure 3) to conditions favoring post-attack aposematism (blue panels) due to a decrease in the influx of naïve predators (decrease in the predator turnover rates). This can be seen in Figure 3B where panels in the lower left corner (low learning speed, low basal detectability) change from gray (no signals) to blue (post-attack

975 signals) as the predator turnover decreases. After post-attack aposematism is established for any reason, the pressure to maintain prey crypticity (i.e. low basal detectability) can be relieved if the strategy provides sufficient protection. In this condition the prey may become more easily detectable, and this trend may be associated with the increase in prey memorability through the heightened basal cognitive cue and the encounter frequency. The increased memorability is a boost to learning speed by itself, and it may also trigger natural selection for faster learning; the cognitive investment may now bring more benefits in foraging efficiency. The increased learning speed may create a situation when the post-attack is no longer the evolutionarily winning strategy. Instead, depending on the ratio of switching penalty to signaling penalty, the pre-attack or permanent aposematism would be favored. In summary, this scenario shows that the post-attack aposematism could open evolutionary pathways to other forms of aposematism. This hypothesis is similar to an existing theory about evolution of aposematism in physically defended animals (Speed \& Ruxton 2005) except that our focus is on behavioral reactions rather than on the physical devices.

\section{Model assumptions and limitations.}


992

993 The above discussions illustrate how our model may contribute to research on diversity of

994 aposematic strategies. However, in order to draw proper generalizations and use the model to

995

996

997

998

999

1000

1001

1002

1003

1004

1005

1006

1007

1008

1009

1010

1011

1012

1013

1014

1015

1016

1017

1018

1019

1020

1021

1022

1023

1024

1025

1026

1027

1028

1029

1030

1031

1032 answer new questions, one needs to understand the model's assumptions and limitations.

First, our model does not feature any coevolution, neither between species nor between traits. Predators can only learn and do not evolve over generations; the prey behavioral strategy is the only trait that has variation for natural selection. In nature, one can expect that there would be predator-prey coevolution as well as the coevolution between behavior, morphology, and physiology. However, proper modeling of such evolutionary interactions would require a number of quantitative assumptions that would limit the applicability and usability of the software. Considering that the signaling behavioral strategy is already a multi-dimensional variable, we found that the further complexity in the modeled world give little benefit while immensely interfering with the visualization, hypothesis formation and the interpretation of reality using the model. Instead, by keeping constant the components that are reasonably expected to evolve slower than prey behavioral strategy, we were able to obtain a complete view across a wide range of conditions. Our primary goal was to generate clear predictions about optimal prey behavioral strategies in a given combination of environmental variables and predator phenotypic and population traits. We realized that modeling coevolution, albeit intriguing, could complicate the accomplishment of this goal.

One omitted variable, however, deserves a more detailed discussion: prey defense. Our model treats prey defense as a given fixed value for each prey individual (repulsive taste), not allowing for variation among prey individuals. It could be seen as an unrealistic assumption because the defensive capability and anti-predatory communication ability is viewed as a closely interacting pair in some systems (Sword et al. 2000; Sherratt 2002; Broom et al. 2005; Speed et al. 2006).

However, allowing the evolution of repulsive taste in our model gives unnecessary complications with minimal benefit. Since the repulsive taste gives one-sided benefit in context of predator deterrence, simply allowing this component to vary is not a proper way of modeling its evolution. It must be accompanied by properly simulated mechanisms of defense cost, which need to bring in an array of assumptions about prey physiology and anatomy, as seen in the theories and discussions introduced above. Furthermore, prey defense can evolve without help of aposematism; chemical, mechanical, or behavioral qualities for antipredator defense could be beneficial in many other contexts as well. This complexity of the real world adds to the difficulty of expanding our model by including defense evolution and the associated mechanisms of costbenefit tradeoffs of defense. Therefore, in our model we decided to set prey defense to a constant value set by the user (repulsive taste value). We hope that the achieved simplicity and universality of the model may be viewed as a strength for asking specific questions that focus on evolution of various signaling strategies.

We also viewed another omitted variable, the common maintenance cost of both the permanent and switchable signals, as a similar unnecessary complication. Unlike the signal 'switchability' maintenance that needs to be balanced against the dynamic benefit of the 
1033 switchability, the static signal maintenance is a common penalty for both switchable and

1034 permanent aposematism. This had lower priority within our primary purpose: our question was

1035 focused on the evolution of the switchable aposematic signals, not the question of aposematism

1036 versus non-aposematism. For readers who are interested in the detailed mechanisms governing

1037 the latter type of evolutionary balance, we would like to recommend other existing theories over

1038 ours; Mappes et al. (2005) and Ruxton et al. (2018) could serve as good entry points.

1039 Finally, for the purpose of this particular paper, we deliberately configured the software

1040 to exclude any startling ('deimatic') or learning facilitation effects of the switched signals. In the

1041 setups used in this paper, the strength of the signal being switched on is the only relevant factor

1042 in predator psychology; the fact that it was switched does not have any effect except that it

1043 imposes some cost to the prey. This decision helped us to study the cost-saving aspects of

1044 switchable aposematism without further complications. However, this assumption is far from

1045 reality (Umbers et al. 2015; Kang et al. 2016; Ruxton et al. 2018), and the model predicts that the

1046 switchable aposematism would be more favored if startle and facilitation effects could provide

1047 survival advantages (Supplementary Figure 1).

\section{Conclusions}

1051

1052

In summary, our simulation provided support for many hypotheses about the diversity of aposematic strategies, especially focused on signal switchability. These include, but are not limited to, the following insights. First, the evolution of pre-attack switchable aposematism may require moderate range of predator learning speed, high basal detectability of the prey, and moderate to high level of signaling penalty. Second, the post-attack switchable aposematism may be favored under relatively low level of predator learning, low prey basal detectability, high signaling penalty. Third, high predator turnover combined with fast learning speed, a condition which produces a mix of naïve and experienced predators, may facilitate evolution of post-attack aposematism. Fourth, higher cost related to signal switching may lead to the disappearance of post-attack aposematism while forcing pre-attack aposematism to be fixed and permanent.

1062 During our study, we developed an individual-based modeling framework that can be used to explore a variety of questions regarding aposematism. This product is capable of simulating a number of variables that are not covered in this article, and we hope that it will inspire scientists and educators to further study the evolutionary biology of aposematic signals. 
1072 We appreciate Keesan Lee of Seoul National University for reviewing the article from a field

1073 biologist's perspective.

1074

1075

1076

1077

References

1078

1079

1080

1081

1082

1083

1084

1085

1086

1087

1088

1089

1090

1091

1092

1093

1094

1095

1096

1097

1098

1099

1100

1101

1102

1103

1104

1105

1106

1107

1108

1109

1110

1111

Bohlin T, Tullberg BS, and Merilaita S. 2008. The effect of signal appearance and distance on detection risk in an aposematic butterfly larva (Parnassius apollo). Animal Behaviour 76:577-584. 10.1016/j.anbehav.2008.02.012

Brandley N, Johnson M, and Johnsen S. 2016. Aposematic signals in North American black widows are more conspicuous to predators than to prey. Behavioral Ecology 27:1104-1112. 10.1093/beheco/arw014

Broom M, Higginson AD, and Ruxton GD. 2010. Optimal investment across different aspects of anti-predator defences. Journal of Theoretical Biology 263:579-586. 10.1016/j.jtbi.2010.01.002

Broom M, Speed MP, and Ruxton GD. 2005. Evolutionarily stable investment in secondary defences. Functional Ecology 19:836-843. 10.1111/j.1365-2435.2005.01030.x

Bura VL, Rohwer VG, Martin PR, and Yack JE. 2011. Whistling in caterpillars (Amorpha juglandis, Bombycoidea): sound-producing mechanism and function. Journal of Experimental Biology 214:30-37. 10.1242/jeb.046805

Crothers L, Gering E, and Cummings M. 2011. Aposematic Signal Variation Predicts Male-Male Interactions in a Polymorphic Poison Frog. Evolution 65:599-605. 10.1111/j.15585646.2010.01154.x

De Cock R, and Matthysen E. 1999. Aposematism and bioluminescence: Experimental evidence from glow-worm Larvae(Coleoptera : Lampyridae). Evolutionary Ecology 13:619-639.

de Solan T, and Aubier TG. 2019. The Evolutionary Importance of Cues in Protective Mimicry. Frontiers in Ecology and Evolution 7. ARTN 283

$10.3389 /$ fevo.2019.00283

Dowdy NJ, and Conner WE. 2016. Acoustic Aposematism and Evasive Action in Select Chemically Defended Arctiine (Lepidoptera: Erebidae) Species: Nonchalant or Not? Plos One 11. ARTN e0152981

10.1371/journal.pone.0152981

Edmunds M. 1972. Defensive Behavior in Ghanaian Praying Mantids. Zoological Journal of the Linnean Society 51:1-\&. DOI 10.1111/j.1096-3642.1972.tb00771.x

Elkins RL. 1984. Taste-Aversion Retention - an Animal-Experiment with Implications for Consummatory-Aversion Alcoholism Treatments. Behaviour Research and Therapy 22:179-186. Doi 10.1016/0005-7967(84)90106-2

Endler JA, and Mappes J. 2004. Predator mixes and the conspicuousness of aposematic signals. American Naturalist 163:532-547. Doi 10.1086/382662 
1112 Gamberale-Stille G, and Guilford T. 2004. Automimicry destabilizes aposematism: predator

1113

1114

1115

1116

1117

1118

1119

1120

1121

1122

1123

1124

1125

1126

1127

1128

1129

1130

1131

1132

1133

1134

1135

1136

1137

1138

1139

1140

1141

1142

1143

1144

1145

1146

1147

1148

1149

1150

1151

1152

1153

1154

1155

1156

1157 sample-and-reject behaviour may provide a solution. Proceedings of the Royal Society BBiological Sciences 271:2621-2625. 10.1098/rspb.2004.2893

Grandcolas P, and Desutter-Grandcolas L. 1998. Successful use of a deimatic display by the praying mantid Polyspilota aeruginosa against the yellow-vented bulbul. Annales De La Societe Entomologique De France 34:335-336.

Grober MS. 1988. Brittle-Star Bioluminescence Functions as an Aposematic Signal to Deter Crustacean Predators. Animal Behaviour 36:493-501. Doi 10.1016/S0003-3472(88)800204

Guilford T. 1994. Go-Slow Signaling and the Problem of Automimicry. Journal of Theoretical Biology 170:311-316. DOI 10.1006/jtbi.1994.1192

Halpin CG, and Rowe C. 2017. The effect of distastefulness and conspicuous coloration on the post-attack rejection behaviour of predators and survival of prey. Biological Journal of the Linnean Society 120:236-244.

Higginson AD, and Ruxton GD. 2009. Dynamic state-dependent modelling predicts optimal usage patterns of responsive defences. Oecologia 160:399-410. 10.1007/s00442-009-1296-y

Kang C, Cho H-J, Lee S-I, and Jablonski PG. 2016. Post-attack aposematic display in prey facilitates predator avoidance learning. Frontiers in Ecology and Evolution 4:35.

Kang CK, Lee SI, and Jablonski PG. 2011. Effect of sex and bright coloration on survival and predator-induced wing damage in an aposematic lantern fly with startle display. Ecological Entomology 36:709-716. 10.1111/j.1365-2311.2011.01319.x

Lariviere S, and Messier F. 1996. Aposematic behaviour in the striped skunk, Mephitis mephitis. Ethology 102:986-992.

Lindstedt C, Huttunen H, Kakko M, and Mappes J. 2011. Disengtangling the evolution of weak warning signals: high detection risk and low production costs of chemical defences in gregarious pine sawfly larvae. Evolutionary Ecology 25:1029-1046. 10.1007/s10682-0109456-4

Lindstedt C, Schroderus E, Lindstrom L, Mappes T, and Mappes J. 2016. Evolutionary constraints of warning signals: A genetic trade-off between the efficacy of larval and adult warning coloration can maintain variation in signal expression. Evolution 70:2562-2572. 10.1111/evo.13066

Lindstrom L, Alatalo RV, Mappes J, Riipi M, and Vertainen L. 1999. Can aposematic signals evolve by gradual change? Nature 397:249-251. Doi 10.1038/16692

Maan ME, and Cummings ME. 2009. Sexual dimorphism and directional sexual selection on aposematic signals in a poison frog. Proceedings of the National Academy of Sciences of the United States of America 106:19072-19077. 10.1073/pnas.0903327106

Mappes J, Kokko H, Ojala K, and Lindstrom L. 2014. Seasonal changes in predator community switch the direction of selection for prey defences. Nature Communications 5. ARTN 5016

$10.1038 /$ ncomms 6016

Mappes J, Marples N, and Endler JA. 2005. The complex business of survival by aposematism. Trends in Ecology \& Evolution 20:598-603. 10.1016/j.tree.2005.07.011

Puurtinen M, and Kaitala V. 2006. Conditions for the spread of conspicuous warning signals: A numerical model with novel insights. Evolution 60:2246-2256.

Robinson MH. 1969. The defensive behaviour of some orthopteroid insects from Panama. Transactions of the Royal Entomological Society of London 121:281-303. 10.1111/j.13652311.1969.tb00521.x

Peer) reviewing PDF | (2019:09:41588:2:0:NEW 2 Mar 2020) 
1158 Ruxton GD, Allen WL, Sherratt TN, and Speed MP. 2018. Avoiding attack : the evolutionary

1159

1160

1161

1162

1163

1164

1165

1166

1167

1168

1169

1170

1171

1172

1173

1174

1175

1176

1177

1178

1179

1180

1181

1182

1183

1184

1185

1186

1187

1188

1189

1190

1191

1192

1193

1194

1195

1196

1197

1198

1199

1200

1201

1202 ecology of crypsis, aposematism, and mimicry. Oxford, United Kingdom: Oxford University Press.

Schlenoff DH. 1985. The Startle Responses of Blue Jays to Catocala (Lepidoptera, Noctuidae) Prey Models. Animal Behaviour 33:1057-1067. Doi 10.1016/S0003-3472(85)80164-0

Sherratt TN. 2002. The coevolution of warning signals. Proceedings of the Royal Society BBiological Sciences 269:741-746. 10.1098/rspb.2001.1944

Sivinski J. 1981. The Nature and Possible Functions of Luminescence in Coleoptera Larvae. The Coleopterists Bulletin 35:167-179.

Skelhorn J, Holmes GG, and Rowe C. 2016. Deimatic or aposematic? Animal Behaviour 113:E1E3. 10.1016/j.anbehav.2015.07.021

Speed MP. 2001. Can receiver psychology explain the evolution of aposematism? Animal Behaviour 61:205-216. 10.1006/anbe.2000.1558

Speed MP, Brockhurst MA, and Ruxton GD. 2010. The Dual Benefits of Aposematism: Predator Avoidance and Enhanced Resource Collection. Evolution 64:1622-1633. 10.1111/j.15585646.2009.00931.x

Speed MP, and Ruxton GD. 2005. Warning displays in spiny animals: One (more) evolutionary route to aposematism. Evolution 59:2499-2508.

Speed MP, Ruxton GD, and Broom M. 2006. Automimicry and the evolution of discrete prey defences. Biological Journal of the Linnean Society 87:393-402. DOI 10.1111/j.10958312.2006.00577.x

Srygley RB. 2004. The aerodynamic costs of warning signals in palatable mimetic butterflies and their distasteful models. Proceedings of the Royal Society B-Biological Sciences 271:589594. 10.1098/rspb.2003.2627

Steinert PA, Infurna RN, and Spear NE. 1980. Long-Term Retention of a Conditioned TasteAversion in Preweanling and Adult-Rats. Animal Learning \& Behavior 8:375-381. Doi 10.3758/Bf03199620

Svadova K, Exnerova A, Stys P, Landova E, Valenta J, Fucikova A, and Socha R. 2009. Role of different colours of aposematic insects in learning, memory and generalization of naive bird predators. Animal Behaviour 77:327-336. 10.1016/j.anbehav.2008.09.034

Sweatt JD. 2009. Mechanisms of memory: Academic Press.

Sword GA, Simpson SJ, El Hadi OTM, and Wilps H. 2000. Density-dependent aposematism in the desert locust. Proceedings of the Royal Society B-Biological Sciences 267:63-68.

Umbers KDL, De Bona S, White TE, Lehtonen J, Mappes J, and Endler JA. 2017. Deimatism: a neglected component of antipredator defence. Biology Letters 13. ARTN 20160936

10.1098/rsbl.2016.0936

Umbers KDL, Fabricant SA, Gawryszewski FM, Seago AE, and Herberstein ME. 2014. Reversible colour change in Arthropoda. Biological Reviews 89:820-848. 10.1111/brv.12079

Umbers KDL, Lehtonen J, and Mappes J. 2015. Deimatic displays. Current Biology 25:R58-R59. Umbers KDL, and Mappes J. 2015. Postattack deimatic display in the mountain katydid, Acripeza reticulata. Animal Behaviour 100:68-73. 10.1016/j.anbehav.2014.11.009

Vallin A, Jakobsson S, Lind J, and Wiklund C. 2005. Prey survival by predator intimidation: an experimental study of peacock butterfly defence against blue tits. Proceedings of the Royal Society B-Biological Sciences 272:1203-1207. 10.1098/rspb.2004.3034

Peer) reviewing PDF | (2019:09:41588:2:0:NEW 2 Mar 2020) 
1203 Wilensky U. 1999. Netlogo. Illinois: Center for Connected Learning and Computer-Based Modeling, Northwestern University.

1205 Williams BL, Brodie ED, and Brodie ED. 2003. Coevolution of deadly toxins and predator resistance: Self-assessment of resistance by garter snakes leads to behavioral rejection of toxic newt prey. Herpetologica 59:155-163.

Doi

$10.1655 / 0018-$ 0831(2003)059[0155:Codtap]2.0.Co;2

1210

Willink B, Brenes-Mora E, Bolanos F, and Prohl H. 2013. Not Everything Is Black and White: Color and Behavioral Variation Reveal a Continuum between Cryptic and Aposematic Strategies in a Polymorphic Poison Frog. Evolution 67:2783-2794. 10.1111/evo.12153

1212 


\section{Table $\mathbf{1}$ (on next page)}

The modeled predator-prey interaction timeline. 


\begin{tabular}{rrrr} 
prey generation & interaction frame & time step & program procedures \\
\hline generation 1 & frame 1 & step 1 & predators-move \\
& & prey-react \\
& prey-pay-cost \\
& predators-search \\
predators-observe \\
& predators-analyze \\
& predators-decide \\
& step 2 & predators-initial-act \\
& predators-feel \\
& prey-react \\
& prey-pay-cost \\
& predators-observe \\
& predators-learn \\
& predators-analyze \\
& predators-decide \\
& & predators-final-act \\
& predators-feel \\
& prey-react \\
& prey-pay-cost \\
& step 3 & predators-observe \\
& &
\end{tabular}

frame 2 (repeat the three time steps for each frame 50 interaction frame)

reproduction stage prey-selectively-die prey-lay

all-prey-die preventextinction predators-turnover prey-emerge

generation 2 $\cdots$ generation 500 (repeat the 50 interaction frames and the reproduction stage for each generation) 
Table 2 (on next page)

The list of model parameters and the values used in this paper. 
parameter

CONTROLLED VARIABLES $\dagger$

world size

generation length

prey frequency

predator frequency

low-signal (L)

high-signal $(\mathrm{H})$

initial attack damage

final attack damage

basal cognitive cue

repulsive taste

behavioral strategies

\section{INDEPENDENT VARIABLES}

switching cost $\uparrow \dagger$

switchability

maintenance cost $\dagger \dagger$

basal detectability

predator turnover

learning speed

signal penalty value(s)

14

50

$92 \%$

$23 \%$

0.3

1

0.1

0.4

0.1

0.1

[NNN, NNL, NNH, NLL, NHH, LLL, LLH, LHH, HHH] “none" (0), "moderate” (0.0005), "high" (0.016)

"none" (0), "moderate" (2.03E-4), "high" (0.00340)

$0.05,0.15,0.4,0.8$

$0.01,0.25,0.7,1$

$0,0.01,0.03,0.1,0.3,1,3,10,30,100,300,1000$

$0.13,0.1105$-- ratio 0.85 , geometric sequence -- 5.32E-5, 4.52E-5, as well as 0

1

$2 \dagger$ Not all controlled variables are explained in the main text, as some of them are not relevant to the 3 questions asked in this paper. For the full description of all simulation parameters, please see

4 Supplementary Materials.

$5 \quad \dagger$ For the purpose of this paper, the two variables were treated as one set under the name 'penalty of

6 switchable signal,' and they changed simultaneously between three states: none, moderate, or high. 


\section{Table 3 (on next page)}

The independent variables used in the paper and the corresponding natural examples. 
2

$\begin{array}{ll}\text { variables } & \begin{array}{l}\text { short } \\ \text { definition }\end{array}\end{array}$

examples (low level)

examples (high level)

COSTS TO PREY

\begin{tabular}{|c|c|c|}
\hline $\begin{array}{l}\text { basal } \\
\text { detectability }\end{array}$ & $\begin{array}{l}\text { basal } \\
\text { discovery } \\
\text { chance when } \\
\text { not giving a } \\
\text { signal }\end{array}$ & $\begin{array}{l}\text { dead leaf mantis } \\
\text { nearly impossible to discover } \\
\text { when not doing threat display }\end{array}$ \\
\hline $\begin{array}{l}\text { signal } \\
\text { penalty }\end{array}$ & $\begin{array}{l}\text { inherent cost } \\
\text { associated to } \\
\text { higher level of } \\
\text { signal }\end{array}$ & $\begin{array}{l}\text { skunk body stripes } \\
\text { there is little evidence of } \\
\text { disadvantage for more striking } \\
\text { bands }\end{array}$ \\
\hline $\begin{array}{l}\text { switching } \\
\text { cost }\end{array}$ & $\begin{array}{l}\text { cost paid per } \\
\text { each } \\
\text { switching } \\
\text { behavior }\end{array}$ & $\begin{array}{l}\text { color under wings } \\
\text { half a wingbeat to expose } \\
\text { pigmentation is very cheap } \\
\text { compared to regular flight }\end{array}$ \\
\hline $\begin{array}{l}\text { switchability } \\
\text { maintenance } \\
\text { cost }\end{array}$ & $\begin{array}{l}\text { cost of } \\
\text { maintaining } \\
\text { the ability to } \\
\text { switch signals }\end{array}$ & $\begin{array}{l}\text { color under wings } \\
\text { if flight is maintained anyway, } \\
\text { there is almost no additional } \\
\text { cost to switchability }\end{array}$ \\
\hline
\end{tabular}

stotting ungulates

their size and habitat often make them

highly noticeable even when not

leaping

\section{black widow spider}

the red dorsal mark is selected to be

moderate because it can be seen by

prey insects (Brandley et al. 2016)

fire-bellied toad unkenreflex the complete flip of the body is a big, energetic movement that may hinder many other useful behavior

\section{rattlesnake rattle}

fragile structure that is kept lifted in locomotion; the tail tip cannot be used in versatile activities like other snakes

\section{PROPERTIES OF PREDATORS}

$\begin{array}{lll}\begin{array}{l}\text { learning } \\ \text { speed }\end{array} & \begin{array}{l}\text { coefficient of } \\ \text { aversive } \\ \text { memory } \\ \text { buildup }\end{array} & \begin{array}{l}\text { mantis-milkweed bug } \\ \text { many intermediate stages } \\ \text { before reaching complete } \\ \text { aversion (Gelperin 1968) }\end{array} \\ \begin{array}{ll}\text { predator } \\ \text { turnover }\end{array} & \begin{array}{l}\text { proportion of } \\ \text { naïve } \\ \text { predators } \\ \text { entering per } \\ \text { prey } \\ \text { generation }\end{array} & \begin{array}{l}\text { blue jay-monarch butterfly } \\ \text { every year only a fraction of } \\ \text { the population needs to learn } \\ \text { about cardenolide }\end{array} \\ & & \end{array}$

primate-snake

aversion acquired quickly and intensely (Ohman \& Mineka 2003)

mantis-milkweed bug probably the whole annual population needs to freshly learn the aversion (Gelperin 1968) 


\section{Figure 1}

The ApoSim model interface built with NetLogo.

The model interface is divided into three parts. In the leftmost panel, the user can adjust model variables using sliders and input windows. In the middle panel, the model status is reported in various plots and displays. In the right panel, the animated view of the twodimensional world is shown.

ApoSim 2.3: Switchable Aposematism

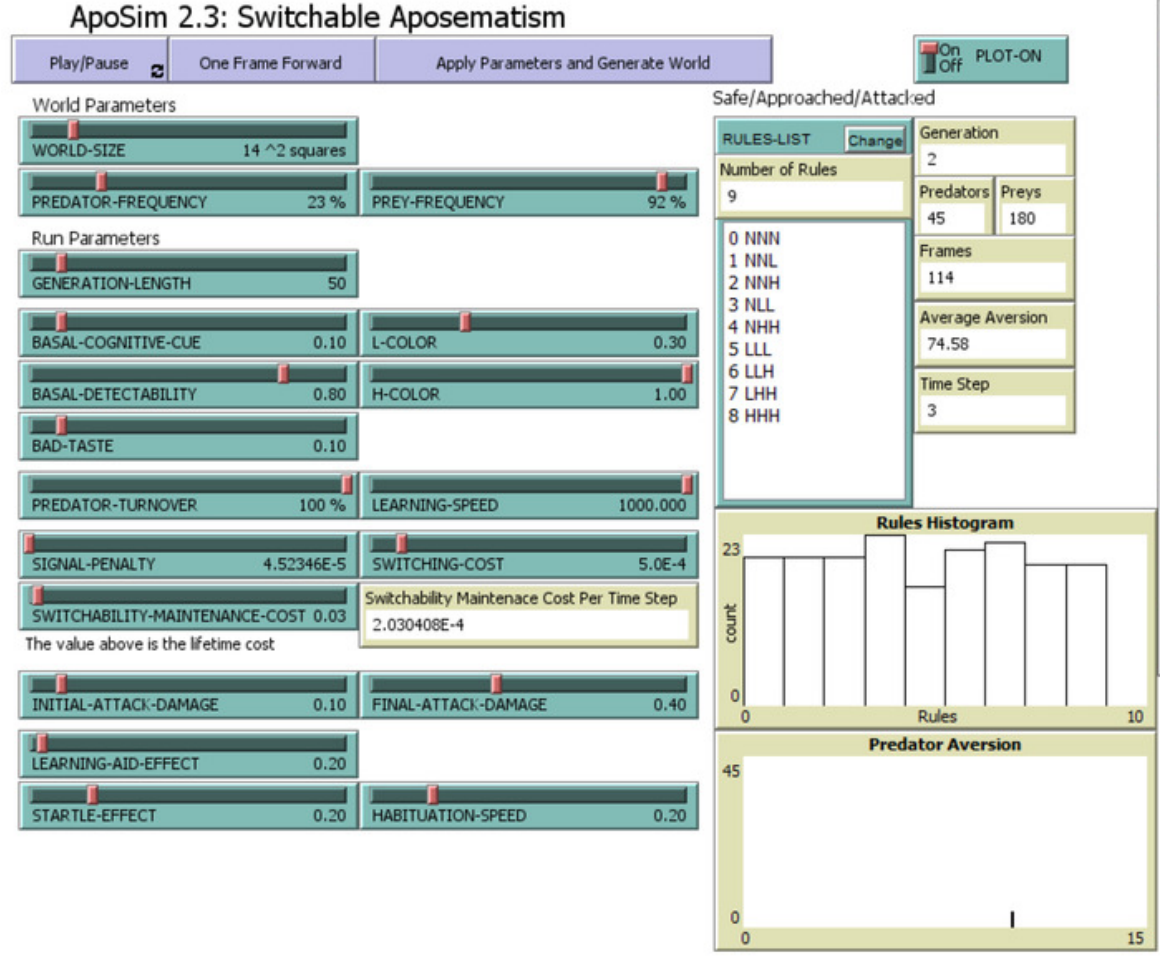

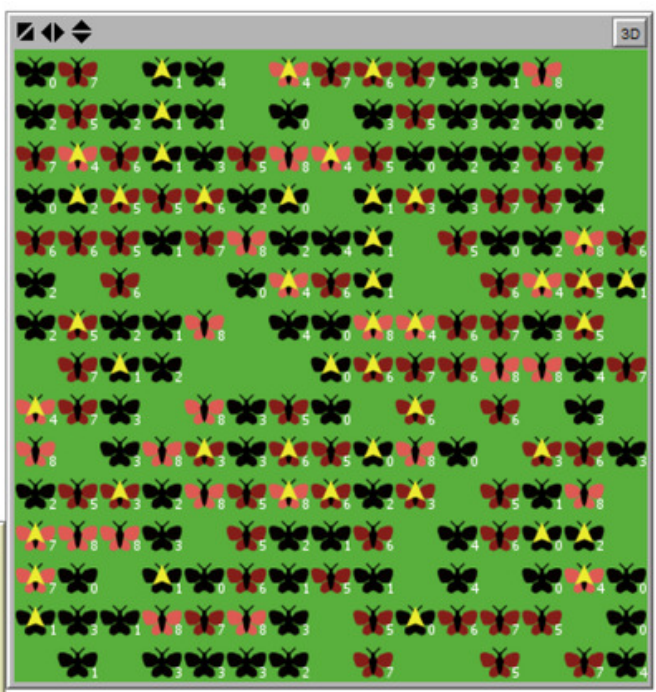



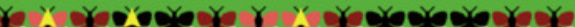
सo

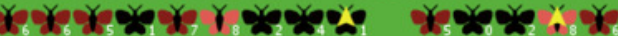

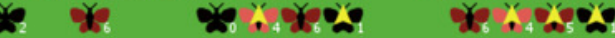

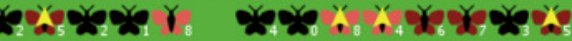

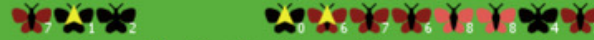



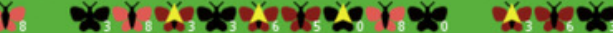

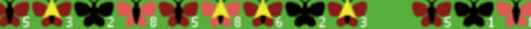

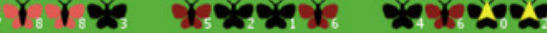








\section{Figure 2}

An example mini-plot of the model results and the color codes for the behavioral strategies.

The prey behavioral strategy that won each run is depicted according to the color code. For the three-letter annotation of the behavioral strategies, see the first section of Methods. The vertical axis is the signal penalty value used in each run. There were three repeated runs for a given combination of conditions, and these repeats appear along the horizontal axis. Therefore, a mini-plot visualizes the result of 153 runs in total, with 51 signal penalty values and 3 repeats. For more detailed information about the run parameters, see Table 2. Mini-plots like the one shown here are the building blocks that constitute the metaplots in Figure 3. 


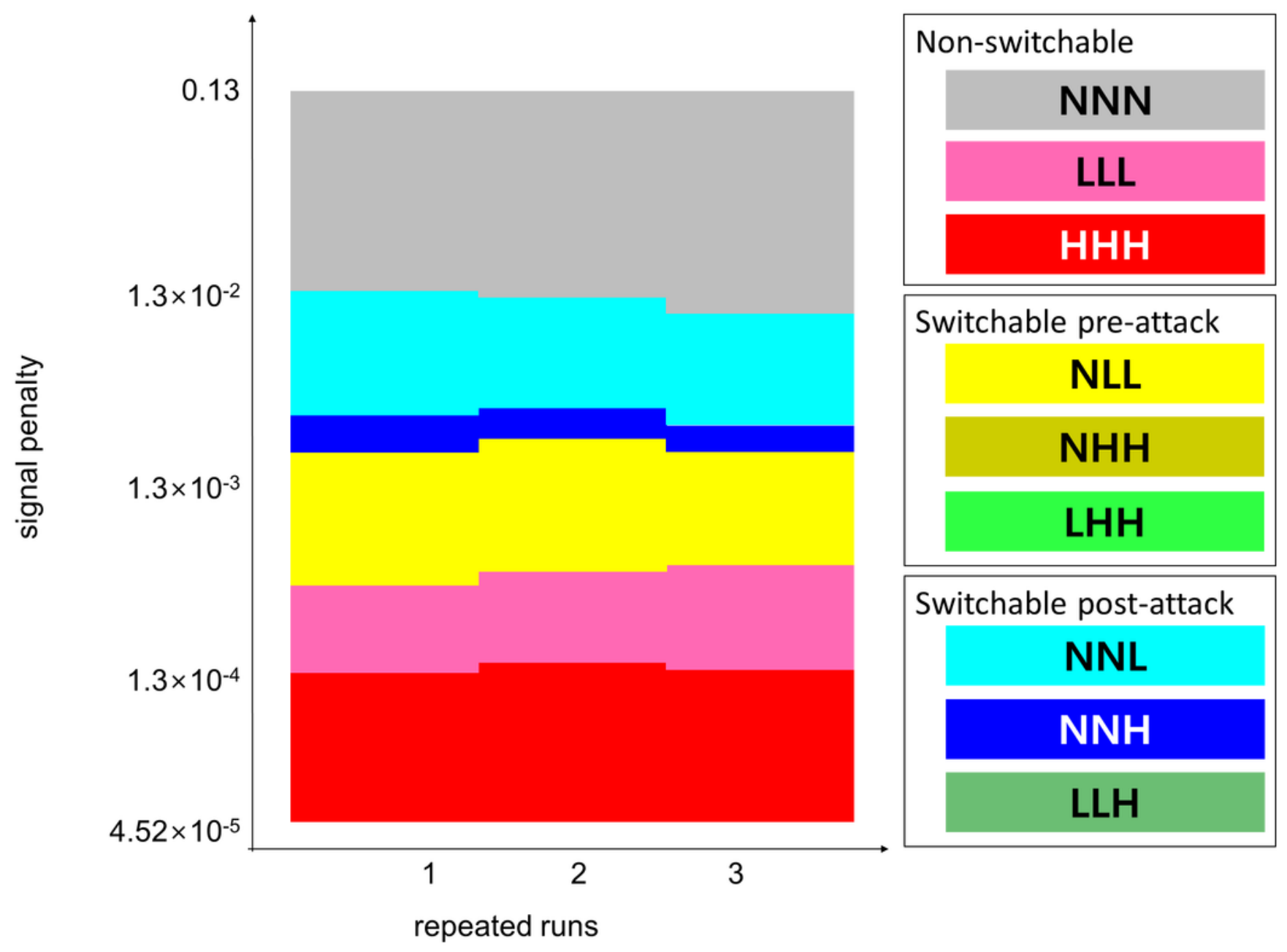




\section{Figure 3}

Model result meta-plots.

The winning behavioral strategies over the entire range of the tested parameters given in Table $\mathbf{2}$. For the color code and the interpretation of each mini-plot, see Figure 2. The x-axis, the one that runs from left to right, is the predator learning speed. The $y$-axis is the predator turnover rate. The $z$-axis is the basal detectability of the prey. For more detailed information about each variable, see Methods section.

(A) the switching cost and the switchability maintenance cost are both none. (B) there are moderate costs for both. (C) the costs are prohibitively high.

For more detail about the three levels of penalty of switchable signals, see Table $\mathbf{2}$. Note that in order to avoid the visual clutter in the three-dimensional view, we labeled each subpanel (A, B, C) with different sets of information; the names of the axes, the regions of interest in lowercase letters, and the values of axes are separately given in subpanel A, B, and C, respectively. However, all notations are applicable to all three subpanels. Regions of interest indicated by small letters $(a-p)$ are referenced in the main text of the Results and Discussion sections. 

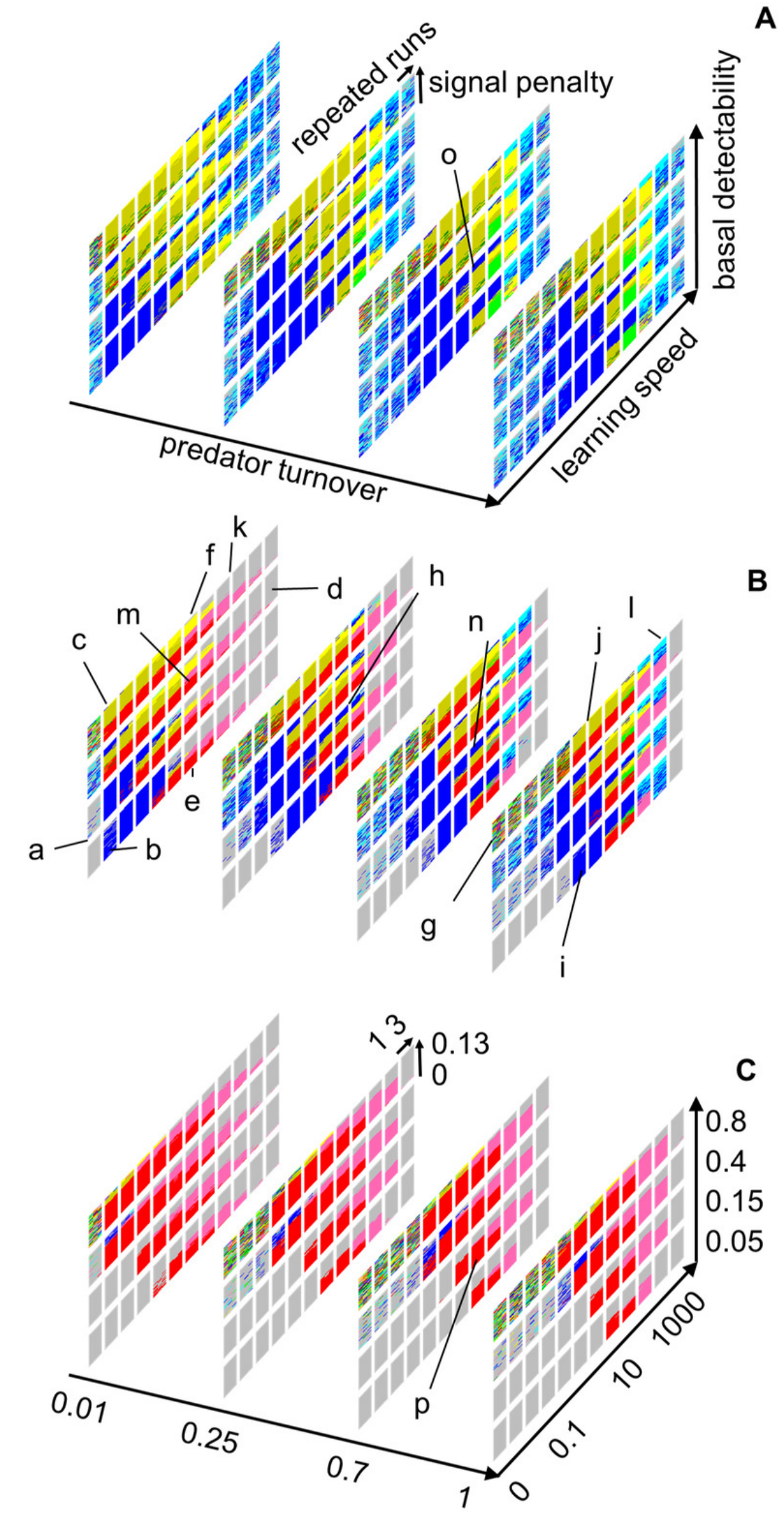

A

\begin{tabular}{|c|c|c|c|c|c|c|c|c|}
\hline \multicolumn{3}{|c|}{ Non-switchable } & \multicolumn{3}{|c|}{ Switchable pre-attack } & \multicolumn{3}{|c|}{ Switchable post-attack } \\
\hline NNN & LLL & HHH & NLL & $\mathrm{NHH}$ & LHH & NNL & NNH & LLH \\
\hline
\end{tabular}




\section{Figure 4}

A two-dimensional diagram for evolutionary stability of permanent versus switchable aposematism.

This is an evolutionary "phase diagram" to abstractly express the stability of the two modes of aposematism. The horizontal axis is the signal penalty, and the vertical axis is the switching penalty (a collective representation of both per-use switching cost and the fixed switchability maintenance cost). The reddish-brown color represents the strength of permanent signals that are expected to be stable in the given condition. The blue color represents the strength of the switchable signals in general, without distinguishing pre- from post-attack switching. Both colors fade as the costs increase indicating that neither permanent nor switchable displays are likely to evolve for extremely high values of both costs. Inlets are mini-plots "n," "o," and "p" of Figure 3, reproduced as examples of three switching penalty levels. For the color codes, axes and ranges of the mini-plots, please see Figure 2 and Figure 3. 


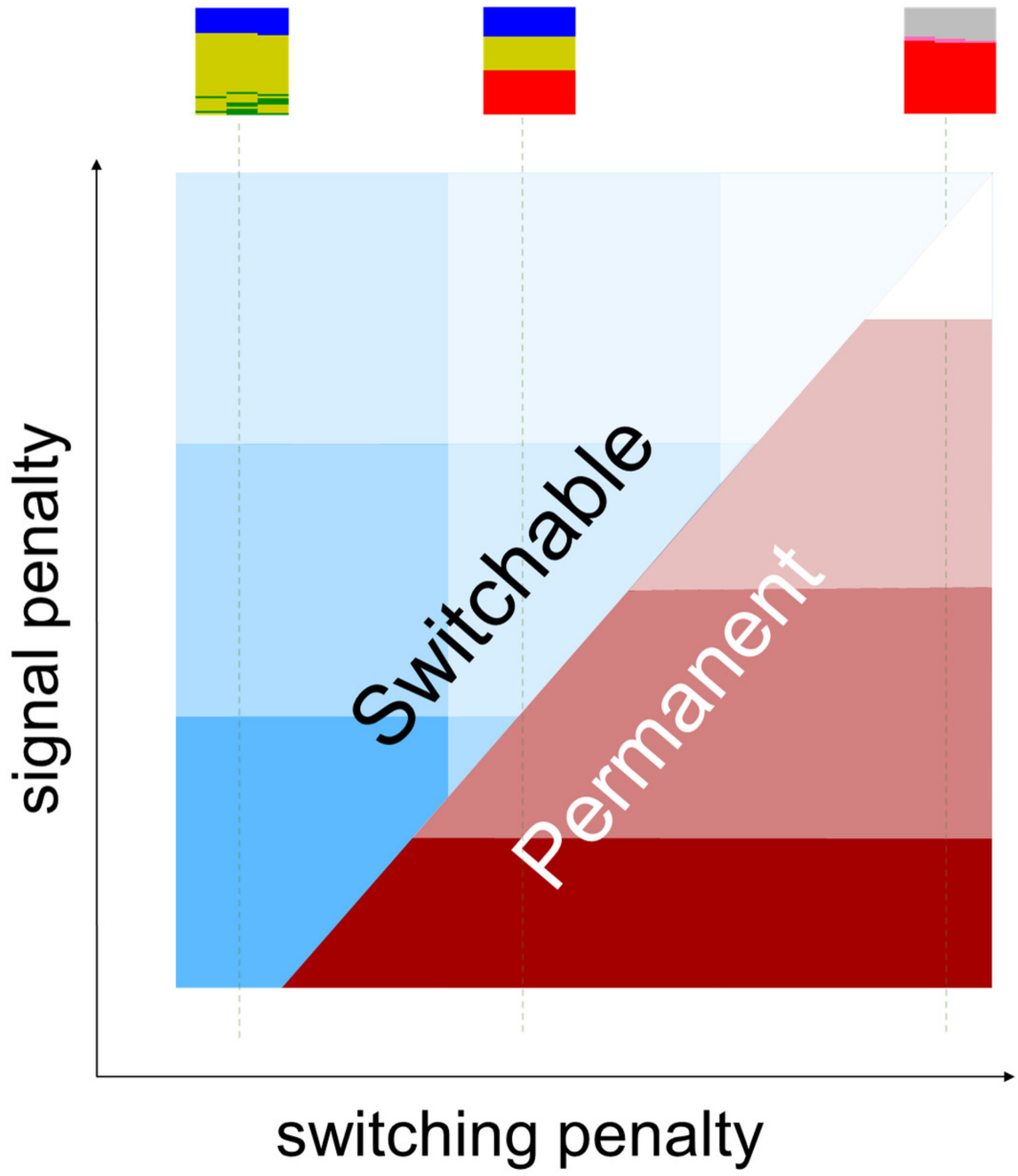




\section{Figure 5}

Three hypothetical pathways towards aposematism.

A portion of the model outcome meta-plot in Figure $\mathbf{3}$ is shown again for explanatory purpose. The labeled arrows $\mathbf{a}, \mathbf{b}$, and $\mathbf{c}$ illustrate the hypothetical pathways, and each is referenced in the main text. These are examples of how the model can be used to discuss hypothetical evolutionary pathways between various forms of (non-)aposematism. Hypothetical mechanisms and ecological conditions critical along such pathways can be identified using the software.



\begin{tabular}{|c|c|c|c|c|c|c|c|c|}
\hline \multicolumn{3}{|c|}{ Non-switchable } & \multicolumn{3}{|c|}{ Switchable pre-attack } & \multicolumn{3}{|c|}{ Switchable post-attack } \\
\hline NNN & LLL & $\mathrm{HHH}$ & NLL & $\mathrm{NHH}$ & LHH & NNL & NNH & LLH \\
\hline
\end{tabular}

